\title{
WHITTLE PSEUDO-MAXIMUM LIKELIHOOD ESTIMATION FOR NONSTATIONARY TIME SERIES*
}

\author{
by \\ Carlos Velasco \\ Universidad Carlos III, Madrid \\ and \\ Peter M Robinson \\ London School of Economics and Political Science
}

Contents:

Abstract

1. Introduction

2. The Model and the Discrete

Fourier Transform

3. Whittle Estimates

4. Simulation Results

5. Illustrative Examples

6. Appendix A: Technical Assumptions and Results

7. Appendix B: Proofs

References

Discussion Paper

No. EM/00/391

May 2000
The Suntory Centre

Suntory and Toyota International Centres for Economics and Related Disciplines London School of Economics and Political Science Houghton Street London WC2A 2AE Tel.: 020-7405 7686

\footnotetext{
* The research of the first author was supported by Spanish Direccion General de Ensenanza Superior, Ref. No. PB98-0025. The second author's research was supported by ESRC Grant No. R000235892, and a Leverhulme Trust Personal Professorship. The authors thank the editor, the associate editor and the referees for very helpful comments.
} 


\begin{abstract}
Whittle pseudo-maximum likelihood estimates of parameters for stationary time series have been found to be consistent and asumptotically normal in the presence of long-range dependence. Generalizing the definition of the memory parameter $d$, we extend these results to include possibly nonstationary $(0.5 \leq d<1)$ or antipersistent $(-0.5<d<0)$ observations. Using adequate data tapers we can apply this estimation technique to any degree of nonstationarity $d \geq 0.5$ without prior knowledge of the memory of the series. We analyse the performance of the estimates on simulated and real data.
\end{abstract}

Keywords: Long-range dependence; nonstationary long memory time series; nonstationary fractional models; frequency domain estimation; tapering.

JEL No.: C22

(C) by the authors. All rights reserved. Short sections of text, not to exceed two paragraphs, may be quoted without explicit permission provided that full credit, including (C) notice, is given to the source. 


\section{Introduction}

Exact and approximate Gaussian maximum likelihood ("Whittle") estimates of parametric stationary time series models have been shown to have the same first-order asymptotic properties under long memory as earlier shown under short memory (e.g. Fox and Taqqu, 1986, Dahlhaus, 1989, Solo, 1989, Giraitis and Surgailis, 1990, Hosoya, 1996). A covariance stationary series with spectral density (sd) $f(\lambda)$ satisfying

$$
f(\lambda) \sim G|\lambda|^{-2 d} \text { as } \lambda \rightarrow 0
$$

where $G>0,|d|<\frac{1}{2}$ and " $\sim$ " means the ratio of left and right sides tends to 1 , is said to have

long memory if $0<d<\frac{1}{2}$, short memory if $d=0$ and negative memory if $-\frac{1}{2}<d<0$.

Nonstationary time series have frequently been assumed to belong to the ARIMA class, such that a finite number of integer differences produces an ARMA short memory process, the degree of differencing being determined by diagnostics such as unit root tests (see Box and Jenkins, 1976). More generally, fractional ARIMA models can be considered such that integer differencing is assumed to produce a series with spectrum satisfying (1), with $d=0$ not assumed. Equivalently, a nonstationary fractional ARIMA series $X_{t}$ is such that $(1-L)^{d} X_{t}$ is a stationary and invertible ARMA, where $d>\frac{1}{2}$ is a real number and $L$ is the lag operator.

Beran (1995) considered a time domain version of Whittle estimation to estimate $d$ along with other parameters in nonstationary fractional ARIMA models. Ling and Li (1997) extended his approach to allow for conditional heteroscedasticity, while Beran, Bhansali and Ocker (1998) discussed model selection in the autoregressive case. We discuss Beran's asymptotic justification below, in view of which we analyze an alternative, discrete-frequency domain version of Whittle. As originally designed for stationary environments (see Hannan, 1973) this of course involves the parameterized sd. However for nonstationary series no sd exists. Nevertheless if $U_{t}^{(s)}=$ $(1-L)^{s} X_{t}, s=\left\lfloor d+\frac{1}{2}\right\rfloor, t>0$, is covariance stationary with mean $\mu$ and $\operatorname{sd} f_{U^{(s)}}(\lambda)$ behaving as $\lambda^{-2(d-s)}$ around $\lambda=0$ we define the pseudo sd (psd) of $X_{t}$ as

$$
f(\lambda):=\left|1-e^{i \lambda}\right|^{-2 s} f_{U^{(s)}}(\lambda) \sim G|\lambda|^{-2 d} \text { as } \lambda \rightarrow 0
$$

Note that if $2 d \geq 1, f(\lambda)$ is not integrable in $[-\pi, \pi]$, is not a sd and cannot represent a decomposition of the (infinite) variance of the nonstationary time series. However, as suggested by Solo (1992) and Hurvich and Ray (1995), the psd $f(\lambda)$ can be interpreted as the limit of the expected sample periodogram, as in the stationary framework. This property was used in Velasco $(1999 \mathrm{a}, \mathrm{b})$ to show that semiparametric narrow-band estimates of the memory parameter $d$ of stationary time series (see Geweke and Porter-Hudak, 1983) have the same desirable asymptotic properties for $d \geq \frac{1}{2}$ as shown by Robinson (1995a, b) for $-\frac{1}{2}<d<\frac{1}{2}$ with tapering needed for large enough $d$ or to eliminate polynomial trends. 


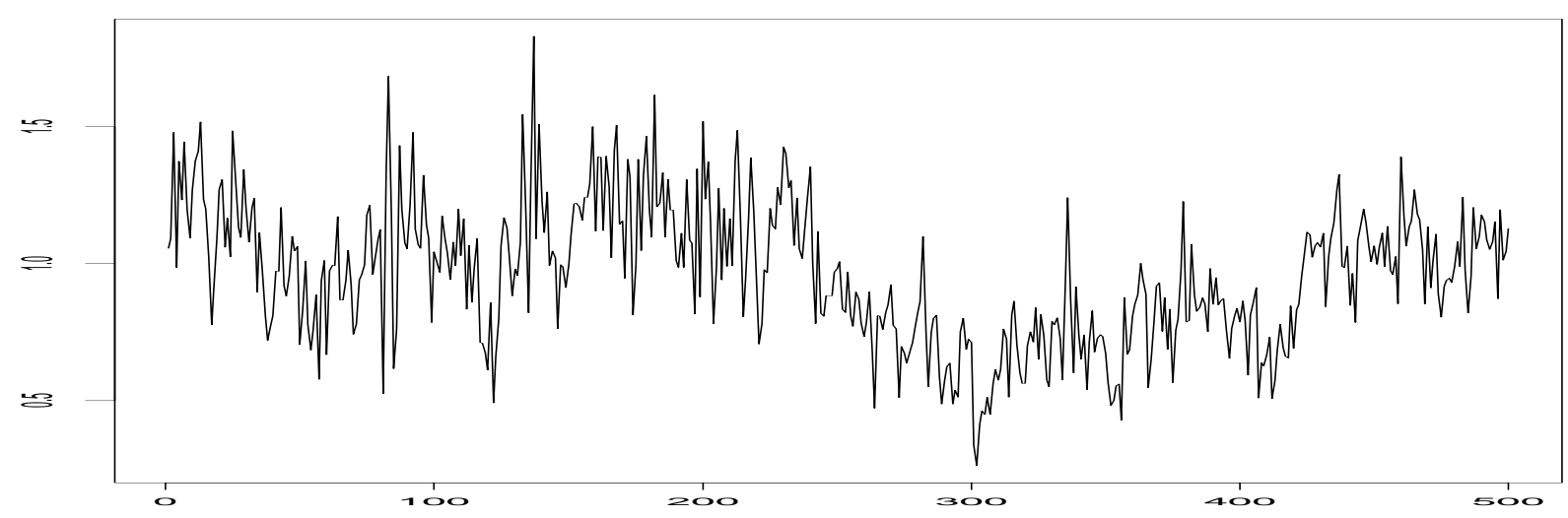

Figure 1: Arizona tree-ring widths (548-1047).

We illustrate the analysis of possibly nonstationary long memory series with the first 500 observations of a series of annual tree-ring widths in Arizona from 548 A.D. onwards obtained by D. A. Graybill in 1984 and maintained by R. Hyndman at www-personal.buseco.monash.edu.au/ hyndman/TSDL. The time plot of Figure 1 shows the prototypical behaviour of long range dependent data with several local trends raising doubts about stationarity. We first analyze this question from a semiparametric point of view and compute Robinson's (1995b) Gaussian semiparametric estimate for bandwidths $m=25$ and 50 with the original and differenced series, adjusting the value of $d$ in the latter case.

Table 1: Memory of tree-ring widths. Semiparametric estimates

\begin{tabular}{lllll}
$\widehat{d}_{\text {Semip }}$ & \multicolumn{3}{c}{$m=50$} \\
\hline$X_{t}$ & .586 & $(.100)$ & .584 & $(.071)$ \\
$\Delta X_{t}$ & .599 & $(.100)$ & .594 & $(.071)$ \\
\hline
\end{tabular}

Though all estimates give values $\widehat{d}>0.5$, confidence intervals based on the asymptotic normal distribution and the standard errors in parentheses include stationary values. These are valid for both stationary and nonstationary series as far as $-0.5<d<0.75$, see Velasco (1999b). We also tried Robinson's (1994b) score tests of stationary and nonstationary hypotheses for fractional exponential $(\operatorname{FEXP}(q))$ models with Bloomfield exponential modeling of short range behaviour (see Section 4). They do not reject the null hypothesis $d=d_{o} \leq 0.5$ comparing onesided statistics to a standard normal for any low order $\operatorname{FEXP}(q)$ model. However for $q \geq 2$ the following t-ratios indicate the presence of some higher level of memory:

Turning to fractional ARIMA modeling, Beran's (1995) estimation procedure selects an AR(3) model with $\widehat{d}=.611$ (.051), while a simpler AR(2) model gives $\widehat{d}=.551$ (.057). These estimates use fractionally differenced data together with a time domain approximation to an ARMA Gaussian likelihood. In fact if we assume that the series is nonstationary and take dif- 
Table 2: Tests of fractional hypothesis for tree-ring widths

\begin{tabular}{lrrrr}
$d_{o}$ & .4 & .5 & .6 & .7 \\
\hline$q=0$ & 1.08 & -2.16 & -4.18 & -5.50 \\
$q=1$ & 1.42 & -1.03 & -2.76 & -3.97 \\
$q=2$ & 4.04 & 1.83 & .14 & -1.13 \\
$q=3$ & 3.87 & 1.99 & .50 & -.66 \\
\hline$q$ is the order of the FEXP $(q)$ model maintained under the null.
\end{tabular}

ferences the results are very close: $\widehat{d}=.627(.051)$ and $\widehat{d}=.564(.057)$ with similar fits for the $\operatorname{AR}(3)$ and $\operatorname{AR}(2)$ parameters.

In the present paper we justify the $\sqrt{n}$-consistency and the use of normal approximations and asymptotic standard errors for the frequency domain (possibly tapered) Whittle estimates of nonstationary series with no prior assumptions on $d$. For the stationary increments of the treering widths the best fit of Whittle estimates is given by an $\operatorname{AR}(2)$ model with $\widehat{d}=.563$. However, working with the original, nonstationary, series and without constraining $\widehat{d}$ to the stationary interval, the same criterion finds that $\widehat{d}=.556$ with almost the same autoregressive parameters. This indicates the claimed robustness to nonstationarity of frequency domain estimation, which could be reinforced by tapering the data if the trending behaviour were very strong. Using the cosine bell taper ((4) below) we confirm the small degree of nonstationarity. We also tried $\operatorname{FEXP}(2)$ models, obtaining similar values of $\widehat{d}$, in agreement with the score tests.

Table 3: Memory of tree-ring widths. Parametric estimates

\begin{tabular}{lllll}
$\hat{d}_{\text {Whittle }}$ & \multicolumn{3}{c}{$\operatorname{AR}(2)$} & FEXP(2) \\
\hline & No taper & Cos taper & No taper & Cos taper \\
\hline$X_{t}$ & $.556(.057)$ & $.536(.082)$ & $.617(.071)$ & $.613(.099)$ \\
$\Delta X_{t}$ & $.563(.057)$ & $.501(.084)$ & $.609(.071)$ & $.574(.099)$ \\
\hline
\end{tabular}

The rest of the paper is organized as follows. The following section presents the parametric model and discusses the properties of the tapered Fourier transform of nonstationary time series. Section 3 defines the Whittle estimates and establishes their asymptotic properties. The finite sample properties of the estimates are examined in a Monte Carlo experiment in Section 4, while Section 5 applies the methods discussed to two empirical series. The technical assumptions and results are summarized in Appendix A, with proofs in Appendix B. 


\section{THE MODEL AND THE DISCRETE FOURIER TRANS- FORM}

We assume that the psd of $X_{t}$ satisfies (2) and belongs to the parametric class defined by

$$
f\left(\lambda ; \sigma^{2}, \theta\right)=\frac{\sigma^{2}}{2 \pi} k(\lambda ; \theta)
$$

where $\theta=\left(\theta^{(1)}, \ldots, \theta^{(a)}\right)^{\prime}$ (with $d=\theta^{(1)}$ ), and $\sigma^{2}$ are any admissible values of the unknown parameter vector $\theta_{o}$ and scalar $\sigma_{o}^{2}$. Thus $f(\lambda)=f\left(\lambda ; \sigma_{o}^{2}, \theta_{o}\right)$. We assume that

$$
\int_{-\pi}^{\pi} \log k(\lambda ; \theta) d \lambda=0, \quad \text { all } \theta
$$

In stationary series, with $d<\frac{1}{2}$, (3) indicates that $\sigma^{2}$, functionally independent of $\theta$, is the variance of the best linear predictor for a process with sd $f\left(\lambda, \sigma^{2}, \theta\right)$. It was employed by Hannan (1973) in his treatment of short memory series and could be relaxed at cost of some extra complexity (see Robinson, 1978, Hosoya and Taniguchi, 1982). However (3) covers standard parameterizations of fractional ARIMA and FEXP models.

Define the tapered discrete Fourier transform (DFT) of $X_{t}$ for $t=1, \ldots, n$, and $\lambda_{j}=2 \pi j / n$, $j$ integer, and a taper sequence $\left\{h_{t}\right\}_{t=1}^{n}$ as

$$
w\left(\lambda_{j}\right)=w\left(X_{t}, h_{t}, \lambda_{j}\right):=\left(2 \pi \sum_{t=1}^{n} h_{t}^{2}\right)^{-1 / 2} \sum_{t=1}^{n} h_{t} X_{t} e^{i \lambda_{j} t}
$$

and the tapered periodogram as $I\left(\lambda_{j}\right)=\left|w\left(\lambda_{j}\right)\right|^{2}$. The usual DFT has $h_{t} \equiv 1$. Typically $h_{t}$ downweights the observations at both extremes of the sequence, leaving largely unchanged the central part of the data.

For short memory processes the untapered periodogram is an inconsistent but asymptotically unbiased estimate at continuity points of the sd and approximately independent across frequencies $\lambda_{j}$. Robinson (1995a) extended such results for long range dependent series, while Velasco (1999a) further extended them to certain nonstationary processes when the memory is not too high, $d<1$, now with respect to the psd (see Appendix A). However the bias and dependence of the periodogram ordinates are affected by sharp peaks in the psd. Tapering was suggested by Tukey (1967) to control leakage problems in spectral estimation when nonstationarity is suspected, as was checked in different frameworks by Zhurbenko (1979), Robinson (1986) and Dahlhaus (1988) among others.

Zhurbenko (1979) used a class of data weights $\left\{h_{t}^{(p)}\right\}$ suggested by Kolmogorov, with $p=$ $1,2, \ldots$, and $N=n / p$ assumed to be integer, proportional to the coefficients $c_{p, N}(t)$ given by

$$
\sum_{t=0}^{p(N-1)} z^{t} c_{p, N}(t+1)=\left(1+z+\cdots+z^{N-1}\right)^{p}=\left(\frac{1-z^{N}}{1-z}\right)^{p} .
$$


These tapers can be obtained by increasingly smooth convolutions of the uniform density (see Alekseev, 1996), and when $p=1$ give the nontapered DFT weights, $h_{t} \equiv 1$, when $p=3$ they are similar to the full cosine bell

$$
h_{t}=\frac{1}{2}\left(1-\cos \frac{2 \pi t}{n}\right),
$$

while when $p=4$ they are very close to Parzen's weights,

$$
h_{t}= \begin{cases}1-6\left[\left|\frac{2 t-n}{n}\right|^{2}-\left|\frac{2 t-n}{n}\right|^{3}\right], & N<t<3 N \\ 2\left\{1-\left|\frac{2 t-n}{n}\right|\right\}^{3}, & 1 \leq t \leq N, \\ & 3 N \leq t \leq 4 N .\end{cases}
$$

The asymptotic properties of the taper sequences depend crucially on the kernel

$$
D_{h}(\lambda):=\sum_{t=1}^{n} h_{t} e^{i \lambda t}
$$

which is the Dirichlet kernel when $h_{t}=1$, and we use them to characterize an extended class of data tapers. We say that a non-negative, symmetric (around $\left.\left\lfloor\frac{n}{2}\right\rfloor\right)$ and normalized $\left(\sup h_{t}=1\right)$ sequence of data tapers $\left\{h_{t}\right\}_{1}^{n}$ is of order $p$ if the following two conditions are satisfied:

1. For $n / p$ integer

$$
D_{h}(\lambda)=\frac{a(\lambda)}{n^{p-1}}\left(\frac{\sin [n \lambda / 2 p]}{\sin [\lambda / 2]}\right)^{p}
$$

where $a(\lambda)$ is a complex function, whose modulus is bounded and bounded away from zero, with $p-1$ derivatives, all bounded for $\lambda \in[-\pi, \pi]$.

2. For some $\gamma_{h}, 0<\gamma_{h} \leq 1, \lim _{n \rightarrow \infty} n^{-1} \sum_{t=1}^{n} h_{t}^{2}=\gamma_{h}$.

Then it can be shown that Parzen weights are of order $p=4$ but cosine bell ones are of order $p=1$, while sharing some properties with tapers of order $p=3$, as discussed in Appendix A. Henceforth when $p=1$ we will imply the usual DFT without tapering and the tapered periodogram with a taper of order $p$ will be denoted as $I^{p}\left(\lambda_{j}\right)$.

As suggested by a referee, summation by parts yields, for a differentiable taper which vanishes at the boundaries, with derivative $h_{t}^{\prime}$,

$$
w\left(X_{t}, h_{t}, \lambda\right) \approx \frac{e^{i \lambda}}{1-e^{i \lambda}}\left[w\left(\Delta X_{t}, h_{t}, \lambda\right)+\frac{w\left(X_{t}, h_{t}^{\prime}, \lambda\right)}{n}\right],
$$

for $\lambda \neq 0(\bmod 2 \pi)$, explaining why a sufficiently smooth taper (i.e. a taper of sufficiently high order $p$ ) can deal with arbitrary levels of memory $d$, justifying also definition (2). In fact, from Hurvich and Ray (1995) and Velasco (1999a), we can obtain Solo's (1992) inversion calculation for $f(\lambda)$ in the nonstationary case,

$$
E\left[I^{p}\left(\lambda_{j p}\right)\right]=\left(2 \pi \sum_{t=1}^{n} h_{t}^{2}\right)^{-1} \int_{-\pi}^{\pi}\left|D_{h}\left(\lambda-\lambda_{j p}\right)\right|^{2} f(\lambda) d \lambda \sim f\left(\lambda_{j p}\right)
$$


as $n \rightarrow \infty$. Then the tapered periodogram can be asymptotically unbiased for the psd $f$ of nonstationary series at Fourier frequencies $\lambda_{j p}, j \neq 0(\bmod N)$, not too close to the origin, though with increased correlation between adjacent ordinates (see Theorems 3 to 5 in Appendix A). Furthermore, using (5) for a data taper of order $p$

$$
w\left(t^{\ell}, h_{t}, \lambda_{j p}\right)=0, \quad \ell=0,1, \ldots, p-1
$$

so tapers also remove polynomial trends in the observed sequence as when e.g. the mean $\mu \neq 0$, if we concentrate on the same set of frequencies $\lambda_{j p}, j \neq 0(\bmod N)$.

\section{WHITTLE ESTIMATES}

To estimate $\theta_{o}$ we use a possibly tapered version of Hannan's (1973) discrete frequency-domain Whittle objective function

$$
Q_{n}(\theta)=\frac{2 \pi p}{n} \sum_{j(p)} \frac{I^{p}\left(\lambda_{j}\right)}{k\left(\lambda_{j} ; \theta\right)}
$$

Here $\sum_{j(p)}$ is a sum over $j=p, 2 p, \ldots, n-p$, assuming for simplicity that $n / p$ is an integer. Thus we omit zero frequency, for mean-correction purposes in the stationary case, while the exclusion of frequencies $\lambda_{j}$ between $\lambda_{p}, \lambda_{2 p}, \ldots, \lambda_{n-p}$ is for (polynomial) trend correction and to guarantee the boundedness of the periodogram expectation under nonstationarity. This $Q_{n}(\theta)$ cannot be replaced by an integral, corresponding to the continuous Whittle objective function, but in any case the discrete form is computationally more convenient and makes more direct use of the fast Fourier transform and functional form for $k(\lambda ; \theta)$. The omission of frequencies when $p>1$ could be avoided to achieve greater efficiency, for example if it is known that $d_{o}<\frac{2}{3}$ and $\mu=0$. As in Hannan (1973) we do not require Gaussianity.

We estimate $\theta_{o}$ by

$$
\widehat{\theta}=\arg \min _{\theta \in \Theta} Q_{n}(\theta)
$$

and estimate $\sigma_{o}^{2}$ by

$$
\widehat{\sigma}^{2}=Q_{n}(\widehat{\theta})
$$

Here $\theta_{o} \in \Theta$, a compact set, and $d_{o} \in \Theta^{(1)}=\left[\nabla_{1}, \nabla_{2}\right]$, a closed interval, $-\frac{1}{2}<\nabla_{1}<\nabla_{2}<$ $\infty$. Thus as in Beran (1995) we exclude noninvertible series. Unlike for the implicitly-defined semiparametric estimators of $d$ (Robinson, 1995b, Velasco, 1999b) there is no restriction on the upper limit $\nabla_{2}$, but the maximum degree of memory $d_{o}$ that we can estimate consistently depends on the tapering applied.

In our statements of Theorems 1 and 2 we refer to assumptions listed in Appendix A.

Theorem 1 Under Assumptions 1 to 4, with $p \geq\left\lfloor d_{o}+\frac{1}{2}\right\rfloor+1$ [only $p>d_{o}$ when $\mu=0$ or $\left.d_{o}<\frac{1}{2}\right], \widehat{\theta} \rightarrow_{p} \theta_{o}$ and $\widehat{\sigma}^{2} \rightarrow_{p} \sigma_{o}^{2}$ as $n \rightarrow \infty$. 
The estimates $\widehat{\theta}$ based on untapered observations $(p=1)$ are consistent for nonstationary observations and any $d_{o}<1$, but only if $\mu=0$, thus covering nonstationary but "mean-reverting" data $\left(\frac{1}{2} \leq d<1\right)$ without drift. When $\mu \neq 0$ we need an increased degree of tapering to eliminate the deterministic trend present in integrated series, $d \geq \frac{1}{2}$, by (6). If $\mu$ is known to be 0 (and there are no other deterministic trends) the tapering required for consistency is the minimum to obtain a periodogram with bounded expectation in (6) when the psd diverges at the origin, $p>d_{o}$. In any case more tapering is needed to obtain asymptotically normally distributed $\widehat{\theta}$.

Depending on the definition of $\Theta$, in the proof we have to consider separately the cases where it is possible that $d \leq d_{o}-\frac{1}{2}$ and those where $\nabla_{1}>d_{o}-\frac{1}{2}$, because of the non-uniform behaviour of $Q_{n}(\theta)$. A similar problem and solution appeared first in Robinson's (1995b) treatment of Gaussian semiparametric estimation for stationary and invertible long memory series with $d_{o} \in$ $\left(-\frac{1}{2}, \frac{1}{2}\right)$. This question also affects Beran's (1995) treatment of nonstationary fractional ARIMA models.

Beran (1995) considered time domain approximate Gaussian maximum likelihood (ML) estimates based on untapered data, whatever the degree of nonstationarity. Of course when stationarity is correctly assumed his estimates are known to have the same asymptotic properties as ours with $p=1$, since only different approximations to the Gaussian likelihood are being employed. For the nonstationary case, Beran's definition of nonstationary processes in effect differs from ours: for the case of a simple fractionally differenced $(0, d, 0)$ model, he considers

$$
(1-L)^{d_{o}} X_{t}=\epsilon_{t}, \quad t>0 ; \quad=0, \quad t \leq 0
$$

where $\epsilon_{t}$ is white noise, whereas we take

$$
\begin{aligned}
(1-L)^{s} X_{t} & =U_{t}, \quad t>0 ; \quad=0, \quad t \leq 0 ; \\
(1-L)^{d_{o}-s} U_{t} & =\epsilon_{t}, \quad t=0, \pm 1, \ldots,
\end{aligned}
$$

for $s=\left\lfloor d_{o}+\frac{1}{2}\right\rfloor$. He considered the objective function $n^{-1} \sum_{t=2}^{n}\left[(1-L)^{d} X_{t}\right]^{2}$. Under (7) this is $n^{-1} \sum_{t=2}^{n}\left[(1-L)^{d-d_{o}} \epsilon_{t}\right]^{2}$, and for consistency one has to consider uniform convergence probability arguments with respect to the whole parameter spaces of admissible $d$, and existence of an asymptotic global minimum at $d=d_{o}$. This involves consideration of the processes $(1-L)^{d-d_{o}} \epsilon_{t}$ which are stationary for $d>d_{o}-\frac{1}{2}$ and nonstationary otherwise. In fact it is the neighbourhood of $d-d_{o}=-\frac{1}{2}$ which causes most difficulty because $(1-L)^{-1 / 2} \epsilon_{t}$ is at the stationary/nonstationary border. Our alternative definition (8) of nonstationary processes, when combined with tapers, avoids this difficulty. The Taylor expansion employed by Beran (1995, p. 670) to prove consistency seems to be circular since the $o_{p}(1)$ error in the expansion for $n^{1 / 2}\left(\widehat{\theta}-\theta_{o}\right)$ is only justified when $\widehat{\theta}$ is in a suitably small neighbourhood of $\theta_{o}$, which presupposes the consistency to be established, while for asymptotic normality of implicitly-defined extremum estimates such as his a rigorous previous proof of consistency is essential. At the same time tapering involves 
Table 4: Bias of $\widehat{d}$ for Fractional $\operatorname{ARIMA}(2, d, 0)$ Models

\begin{tabular}{|c|c|c|c|c|c|c|c|c|c|c|c|c|c|}
\hline & & \multicolumn{6}{|c|}{$n=512$} & \multicolumn{6}{|c|}{$n=200$} \\
\hline$d$ & & -.4 & .4 & .6 & .9 & 1.1 & 1.4 & -.4 & .4 & .6 & .9 & 1.1 & 1.4 \\
\hline \multicolumn{14}{|c|}{ No taper, $p=1$} \\
\hline \multirow[t]{4}{*}{$\widehat{d}$} & G-SEM & -.037 & -.041 & -.036 & -.023 & -.078 & -.350 & -.206 & -.105 & -.267 & -.246 & -.225 & -.362 \\
\hline & $\mathrm{W}-\mathrm{p}$ & -.006 & .002 & .002 & -.025 & -.137 & -.395 & -.023 & .070 & -.095 & -.081 & -.067 & -.456 \\
\hline & $\mathrm{W}-2 \mathrm{~S}$ & -.017 & .002 & -.003 & .000 & -.027 & -.014 & -.033 & .116 & -.034 & -.037 & -.037 & -.073 \\
\hline & ML-2S & -.002 & .004 & .004 & -.004 & -.028 & -.030 & -.012 & .128 & -.022 & -.020 & -.016 & -.109 \\
\hline \multicolumn{14}{|c|}{ Taper, $p=2$} \\
\hline \multirow[t]{4}{*}{$\widehat{d}$} & G-SEM & -.068 & -.064 & -.059 & -.048 & -.059 & .007 & -.270 & -.261 & -.253 & -.237 & -.222 & -.188 \\
\hline & $\mathrm{W}-\mathrm{p}$ & -.037 & -.026 & -.023 & -.018 & .006 & .017 & -.082 & -.076 & -.071 & -.061 & -.050 & -.029 \\
\hline & $\mathrm{W}-2 \mathrm{~S}$ & -.018 & -.009 & -.013 & -.016 & -.007 & .009 & -.035 & -.031 & -.033 & -.036 & -.037 & -.031 \\
\hline & $\mathrm{ML}-2 \mathrm{~S}$ & -.006 & .007 & .005 & .007 & .020 & .030 & -.014 & -.010 & -.011 & -.009 & -.004 & .013 \\
\hline
\end{tabular}

an efficiency loss (see Theorem 2 below), and Beran's simulations support his insight that ML estimates of fractional models have the classical $\sqrt{n}$-consistency, asymptotic normality and efficiency properties. Indeed, it is consistent with Robinson's (1994b) findings that score tests for a unit root and many other stationary and nonstationary null hypotheses, when directed against fractional alternatives such as (7), have standard asymptotics, since the test statistic depends on only the null differenced data. By contrast unit root tests against autoregressive alternatives have nonstandard asymptotics (see e.g. Solo, 1984).

We now discuss the asymptotic normality of $\widehat{\theta}$.

Theorem 2 Under Assumptions 1 to 4,7 to 9 and

(i) Assumption 5 if $p=1$ ( $\mu=0$ or $\left.d_{o}<\frac{1}{2}\right)$, with $d_{o}<\frac{3}{4}$;

(ii) Assumption 6 if $p>1$ (any $\mu$ ), such that $p \geq\left\lfloor d_{o}+\frac{1}{2}\right\rfloor+1, \beta>1$, as $n \rightarrow \infty$

$$
\sqrt{n}\left(\widehat{\theta}-\theta_{o}\right) \rightarrow_{d} N\left(0,4 \pi p \Phi_{p} \Sigma_{o}^{-1}\right)
$$

The asymptotic variance formula is the same as for stationary series, with

$$
\Sigma_{o}=\int_{-\pi}^{\pi}\left\{\frac{\partial}{\partial \theta} \log k\left(\lambda ; \theta_{o}\right)\right\}\left\{\frac{\partial}{\partial \theta^{\prime}} \log k\left(\lambda ; \theta_{o}\right)\right\} d \lambda
$$

and it may be shown that

$$
\frac{2 \pi}{n} \sum_{j=1}^{n-1}\left\{\frac{\partial}{\partial \theta} \log k\left(\lambda_{j} ; \widehat{\theta}\right)\right\}\left\{\frac{\partial}{\partial \theta^{\prime}} \log k\left(\lambda_{j} ; \widehat{\theta}\right)\right\}
$$

is a consistent estimate of $\Sigma_{o}$. $\Phi_{p}$ is the taper variance inflation factor, $\Phi_{p}=\lim _{n \rightarrow \infty} \sum_{k=p, 2 p, \ldots}^{n}$ $h^{2}\left(\lambda_{k}\right)$ with $h(\lambda)=\left(\sum_{1}^{n} h_{t}^{2}\right)^{-1} \sum_{1}^{n} h_{t}^{2} \cos t \lambda$, which takes the values $1.05000,1.00354$ and 1.00086 
Table 5: Standard deviation of $\widehat{d}$ for Fractional ARIMA(2,d,0) Models

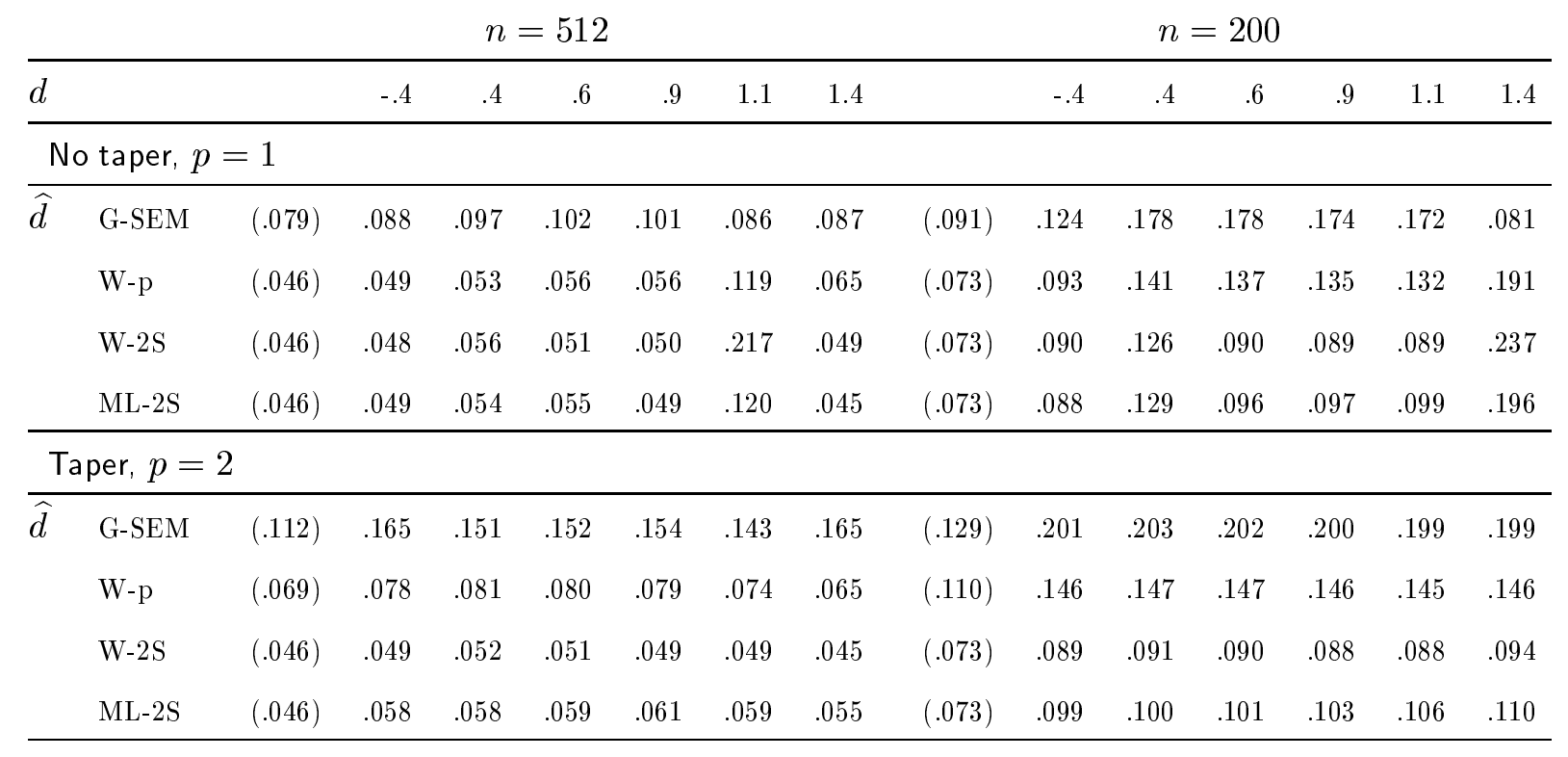

for the Zhurbenko data tapers with $p=2,3,4$ respectively, implying modest increments of the variance of $5 \%, .35 \%$ and $.09 \%$ for each of the data tapers (apart from the extra factor $p$ due to the sampling of frequencies). Note that if we summed for $k=1,2, \ldots, n$ in $\Phi_{p}$ by considering all Fourier frequencies in $Q_{n}$, then by Parseval's identity $\Phi_{p}=\lim _{n \rightarrow \infty} n\left(\sum h_{t}^{2}\right)^{-2} \sum h_{t}^{4}$ would be the usual tapering variance correction (see e.g. Dahlhaus, 1985) and $\Phi_{1}=1$ by orthogonality of the sine and cosine functions.

The same result holds for the cosine bell taper (4) when $d_{o}<\frac{3}{2}$ and $\mu=0$ (or $d_{o}<\frac{1}{2}$ for any $\mu$ ) are known, where in this case it is possible to include all frequencies $\lambda_{j}, 2 \leq j \leq n-2$, in $Q_{n}$ as if actually $p=1$, obtaining $\Phi_{\cos }=\frac{35}{18}$. This accounts for a reduction of the variance of over $33 \%$ from setting $p$ as 3 , involving then only asymptotically uncorrelated periodogram ordinates in $Q_{n}$ (see Theorem 4 in Appendix A).

In fractional models, because of the separate modeling of short and long run behaviour, $\theta=\left(d, \theta^{(-1)}\right)^{\prime}, f\left(\lambda ; \sigma^{2}, \theta\right)=\left(\sigma^{2} /(2 \pi)\right)\left|1-e^{i \lambda}\right|^{-2 d} h\left(\lambda ; \theta^{(-1)}\right)$, where $h$ is a short memory sd, corresponding, for example, to an ARMA or Bloomfield (1973) exponential model (see (9) below), the asymptotic variance of the parameter estimates is free of $d_{o}$, and therefore of the degree of nonstationarity of the observed time series (apart from the effects of tapering if used), and again is consistent with the nature of the score tests of Robinson (1994b). Initial differencing improves asymptotic efficiency only if a lower order taper is used (with smaller $p$, since the contribution of $\Phi_{p}$ is of less significance), but this makes all estimates more sensitive to peaks or nonstationarity at other frequencies (see the conclusions of Hauser (1999) for stationary fractional ARIMA models and various methods of approximate ML estimation, including tapered-Whittle estimates). In any case, the steeper $f(\lambda)$ at $\lambda=0$, i.e. the larger $d$, the worse the asymptotic approximations 
Table 6: Bias of $\widehat{\phi}_{1}$ and $\widehat{\phi}_{2}$ for Fractional $\operatorname{ARIMA}(2, d, 0)$ Models

\begin{tabular}{|c|c|c|c|c|c|c|c|c|c|c|c|c|c|}
\hline \multirow{2}{*}{$\frac{}{d}$} & & \multicolumn{6}{|c|}{$n=512$} & \multicolumn{6}{|c|}{$n=200$} \\
\hline & & -.4 & .4 & .6 & .9 & 1.1 & 1.4 & -.4 & .4 & .6 & .9 & 1.1 & 1.4 \\
\hline \multicolumn{14}{|c|}{ No taper, $p=1$} \\
\hline \multirow[t]{3}{*}{$\widehat{\phi}_{1}$} & W-p & -.004 & -.004 & -.005 & -.081 & -.316 & -.601 & .011 & .038 & .047 & .041 & .035 & -.503 \\
\hline & $\mathrm{W}-2 \mathrm{~S}$ & .001 & -.003 & .001 & -.004 & .003 & .003 & .015 & .014 & .014 & .014 & .014 & .019 \\
\hline & ML-2S & -.007 & -.003 & -.001 & .000 & .006 & .015 & .004 & .009 & .009 & .008 & .005 & .064 \\
\hline \multirow[t]{3}{*}{$\widehat{\phi}_{2}$} & $\mathrm{~W}-\mathrm{p}$ & .003 & .006 & .001 & .112 & .390 & .585 & .008 & .004 & .003 & .004 & .005 & .575 \\
\hline & $\mathrm{W}-2 \mathrm{~S}$ & .002 & .005 & -.004 & .005 & .002 & .002 & .007 & .007 & .007 & .006 & .006 & .026 \\
\hline & ML-2S & .000 & .002 & -.008 & .002 & .000 & -.002 & .002 & .002 & .002 & .001 & .001 & .009 \\
\hline \multicolumn{14}{|c|}{ Taper, $p=2$} \\
\hline \multirow[t]{3}{*}{$\overline{\widehat{\phi}_{1}}$} & $\mathrm{~W}-\mathrm{p}$ & .010 & .014 & .013 & .010 & .002 & .048 & .042 & .040 & .038 & .033 & .029 & .019 \\
\hline & $\mathrm{W}-2 \mathrm{~S}$ & .001 & .003 & .005 & .006 & .002 & .015 & .017 & .013 & .013 & .014 & .014 & .010 \\
\hline & ML-2S & -.005 & -.005 & -.004 & -.005 & -.008 & .037 & .005 & .003 & .003 & .002 & -.001 & -.010 \\
\hline \multirow[t]{3}{*}{$\widehat{\phi}_{2}$} & W-p & -.001 & -.006 & -.005 & -.005 & .000 & .009 & .000 & .001 & .001 & .002 & .004 & .008 \\
\hline & $\mathrm{W}-2 \mathrm{~S}$ & .002 & .000 & -.001 & -.001 & .002 & .027 & .007 & .008 & .007 & .006 & .006 & .010 \\
\hline & ML-2S & .000 & -.003 & -.004 & -.004 & -.001 & .011 & .002 & .002 & .001 & .001 & .001 & .003 \\
\hline
\end{tabular}

can be expected for finite samples.

\section{SIMULATION RESULTS}

In this section we investigate the performance of Whittle estimates for simulated stationary and nonstationary data. We generated independent samples of two Gaussian time series models with several values of the memory parameter $d$ and two sample sizes $(n=200$ and 512 , with 1000 and 100 replications respectively). The short memory components are $\operatorname{ARMA}(2,0)$ with autoregressive parameters $\phi_{1}=0.65, \phi_{2}=-0.6$ and $\sigma=4$, and Bloomfield's (1973) exponential model as proposed in Robinson (1994a, p. 73), with parameters $\sigma^{2}=2 \pi e^{-1}$ (which corresponds to $\theta_{0}=-1$ in the usual parameterization), $\theta_{1}=5$ and $\theta_{2}=-3$, leading to the $\operatorname{FEXP}(2)$ model

$$
f\left(\lambda ; \sigma^{2}, \theta\right)=\frac{\sigma^{2}}{2 \pi}\left|1-e^{i \lambda}\right|^{-2 d} e^{\theta_{1} \cos \lambda+\theta_{2} \cos 2 \lambda} .
$$

These models have variances of similar order of magnitude and psd of similar shape, with a peak around $\pi / 3$ and comparable behaviour at the origin. The memory parameters used were $d=-0.4$ to analyze series close to non-invertibility, $d=0.4$, to compare with the stationary case, $d=0.6,0.9$, nonstationary but still mean-reverting series, and $d=1.1,1.4$, difference stationary $(\mu=0)$. 
Table 7: Standard deviation of $\widehat{\phi}_{1}$ and $\widehat{\phi}_{2}$ for Fractional $\operatorname{ARIMA}(2, d, 0)$ Models

\begin{tabular}{|c|c|c|c|c|c|c|c|c|c|c|c|c|c|c|c|}
\hline \multirow{2}{*}{$\frac{}{d}$} & & \multicolumn{7}{|c|}{$n=512$} & \multicolumn{7}{|c|}{$n=200$} \\
\hline & & & -.4 & .4 & .6 & .9 & 1.1 & 1.4 & & -.4 & .4 & .6 & .9 & 1.1 & 1.4 \\
\hline \multicolumn{16}{|c|}{ No taper, $p=1$} \\
\hline \multirow[t]{3}{*}{$\widehat{\phi}_{1}$} & W-p & $(.044)$ & .043 & .047 & .043 & .098 & .187 & .088 & $(.071)$ & .078 & .101 & .107 & .106 & .106 & .204 \\
\hline & $\mathrm{W}-2 \mathrm{~S}$ & $(.044)$ & .043 & .048 & .041 & .049 & .043 & .046 & $(.071)$ & .078 & .077 & .077 & .077 & .077 & .102 \\
\hline & ML-2S & $(.044)$ & .043 & .048 & .043 & .048 & .042 & .044 & $(.071)$ & .078 & .079 & .080 & .081 & .081 & .147 \\
\hline \multirow[t]{3}{*}{$\widehat{\phi}_{2}$} & W-p & $(.036)$ & .036 & .039 & .037 & .123 & .188 & .065 & $(.057)$ & .058 & .075 & .081 & .081 & .081 & .115 \\
\hline & $\mathrm{W}-2 \mathrm{~S}$ & $(.036)$ & .036 & .038 & .036 & .038 & .036 & .031 & $(.057)$ & .058 & .059 & .059 & .059 & .059 & .103 \\
\hline & ML-2S & $(.036)$ & .035 & .038 & .036 & .037 & .035 & .030 & $(.057)$ & .058 & .058 & .058 & .058 & .058 & .061 \\
\hline \multicolumn{16}{|c|}{ Taper, $p=2$} \\
\hline \multirow[t]{3}{*}{${\widehat{\phi_{1}}}_{1}$} & W-p & $(.066)$ & .063 & .071 & .070 & .070 & .063 & .215 & $(.106)$ & .115 & .115 & .115 & .115 & .114 & .115 \\
\hline & $\mathrm{W}-2 \mathrm{~S}$ & $(.044)$ & .043 & .046 & .046 & .045 & .043 & .197 & $(.071)$ & .078 & .077 & .077 & .076 & .077 & .078 \\
\hline & ML-2S & $(.044)$ & .045 & .049 & .049 & .050 & .046 & .190 & $(.071)$ & .082 & .083 & .083 & .084 & .085 & .086 \\
\hline \multirow[t]{3}{*}{$\widehat{\phi}_{2}$} & $\mathrm{~W}-\mathrm{p}$ & $(.054)$ & .054 & .050 & .050 & .050 & .054 & .061 & $(.085)$ & .082 & .082 & .082 & .082 & .082 & .083 \\
\hline & $\mathrm{W}-2 \mathrm{~S}$ & $(.036)$ & .036 & .031 & .031 & .031 & .036 & .128 & $(.057)$ & .058 & .059 & .059 & .059 & .059 & .060 \\
\hline & ML-2S & $(.036)$ & .035 & .031 & .030 & .030 & .035 & .058 & $(.057)$ & .058 & .058 & .058 & .058 & .058 & .058 \\
\hline
\end{tabular}

Stationary ARFIMA series were simulated by the S-PLUS function arima.fracdiff.sim and then integrated an integer number of times if $d \geq 0.5$. For the exponential models (9), we simulated first the short memory model with $d=0$ and then integrated fractionally to reach the appropriate value of $d$. For the short memory simulation of the exponential models we used the Davies and Harte (1987) algorithm, as programmed by Beran (1994), using the first 50 autocovariances obtained by numerical integration of the sd.

Non-tapered ( $p=1$ ) and tapered (with Zhurbenko taper of order $p=2$ ) data were considered. Also the cosine bell taper (4) was used but the results were similar to the taper with $p=2$ and are not reported here. The estimates compared are the following:

1. G-SEM: Gaussian semiparametric narrow-band estimate of $d$ (Robinson, 1995b, Velasco, 1999 b) with bandwidth numbers $m=30$ (20 for the FEXP model) and 40 for each sample size.

2. W-p: Whittle estimates $\widehat{\theta}$.

These estimates are consistent and asymptotically normal for all $d$ we tried when tapering is applied, but only consistent for $d<1$, and asymptotically normal for $d<\frac{3}{4}$ if the raw series is used. Using the Whittle memory estimates $\widehat{\theta}_{1}=\widehat{d}(p)$ from $\mathrm{W}-\mathrm{p}$, it is possible to fractionally difference $X_{t}$ to achieve approximate short memory stationarity and then use standard untapered 
Table 8: Bias of $\widehat{d}$ for Bloomfield FEXP(2) Models

\begin{tabular}{|c|c|c|c|c|c|c|c|c|c|c|c|c|c|}
\hline & & \multicolumn{6}{|c|}{$n=512$} & \multicolumn{6}{|c|}{$n=200$} \\
\hline$d$ & & -.4 & .4 & .6 & .9 & 1.1 & 1.4 & -.4 & .4 & .6 & .9 & 1.1 & 1.4 \\
\hline \multicolumn{14}{|c|}{ No taper, $p=1$} \\
\hline \multirow[t]{3}{*}{$\widehat{d}$} & G-SEM & -.072 & .169 & .258 & .086 & -.099 & -.396 & -.150 & -.191 & -.175 & -.144 & -.480 & -.373 \\
\hline & W-p & -.149 & -.009 & .093 & .026 & -.137 & -.426 & -.259 & -.359 & -.379 & -.397 & -.623 & -.411 \\
\hline & $\mathrm{W}-2 \mathrm{~S}$ & -.175 & -.121 & -.156 & -.208 & -.215 & -.020 & -.313 & -.361 & -.386 & -.380 & -.639 & -.362 \\
\hline \multicolumn{14}{|c|}{ Taper, $p=2$} \\
\hline \multirow[t]{3}{*}{$\widehat{d}$} & G-SEM & -.130 & -.117 & -.108 & -.099 & -.093 & -.079 & -.242 & -.253 & -.215 & -.205 & -.179 & -.149 \\
\hline & $\mathrm{W}-\mathrm{p}$ & -.074 & -.064 & -.056 & -.053 & -.085 & -.121 & -.216 & -.225 & -.203 & -.208 & -.239 & -.335 \\
\hline & $\mathrm{W}-2 \mathrm{~S}$ & .546 & -.054 & -.041 & -.101 & -.135 & -.221 & .365 & -.202 & -.214 & -.144 & -.176 & -.374 \\
\hline
\end{tabular}

stationary long memory methods to evaluate the first Whittle step in 2., which uses possibly tapered nonstationary inputs. We propose two alternative two-step (asymptotically equivalent) procedures, where the second step's input is in both cases the untapered $\Delta^{\widehat{d}(p)} X_{t}$ :

3. W-2S: Two-Step Whittle estimates, where the second step is Whittle (stationary) estimation, $p=1$.

4. ML-2S: Two-Step time domain (stationary) ML estimates for ARIMA stationary series, where the second step is implemented by the S-PLUS function arima.fracdiff.mle (see Haslett and Raftery, 1989).

All parametric estimates (2. through 4.) use the same (known) true model, since otherwise the estimates of $d$ are not guaranteed consistent for the second step (even if enough tapering were applied), and tapering is only used for the first step estimates, since it is hoped that differencing achieves stationarity of the second step inputs.

For Whittle estimates (and the Gaussian semiparametric), the minimum of $Q_{n}$ was found using the S-PLUS function nlmin. We report bias and standard error across replications. The asymptotic standard deviations for each particular sample size are in parentheses, taking into account the tapering applied and assuming that the two-step estimates have the ML asymptotic variance.

\subsection{ARFIMA models}

The summary of the simulations is contained in Tables 4 and 5 for the estimates of $d$ and in Tables 6 and 7 for the estimates of $\phi_{1}$ and $\phi_{2}$. 
Table 9: Standard deviation of $\widehat{d}$ for Bloomfield FEXP(2) Models

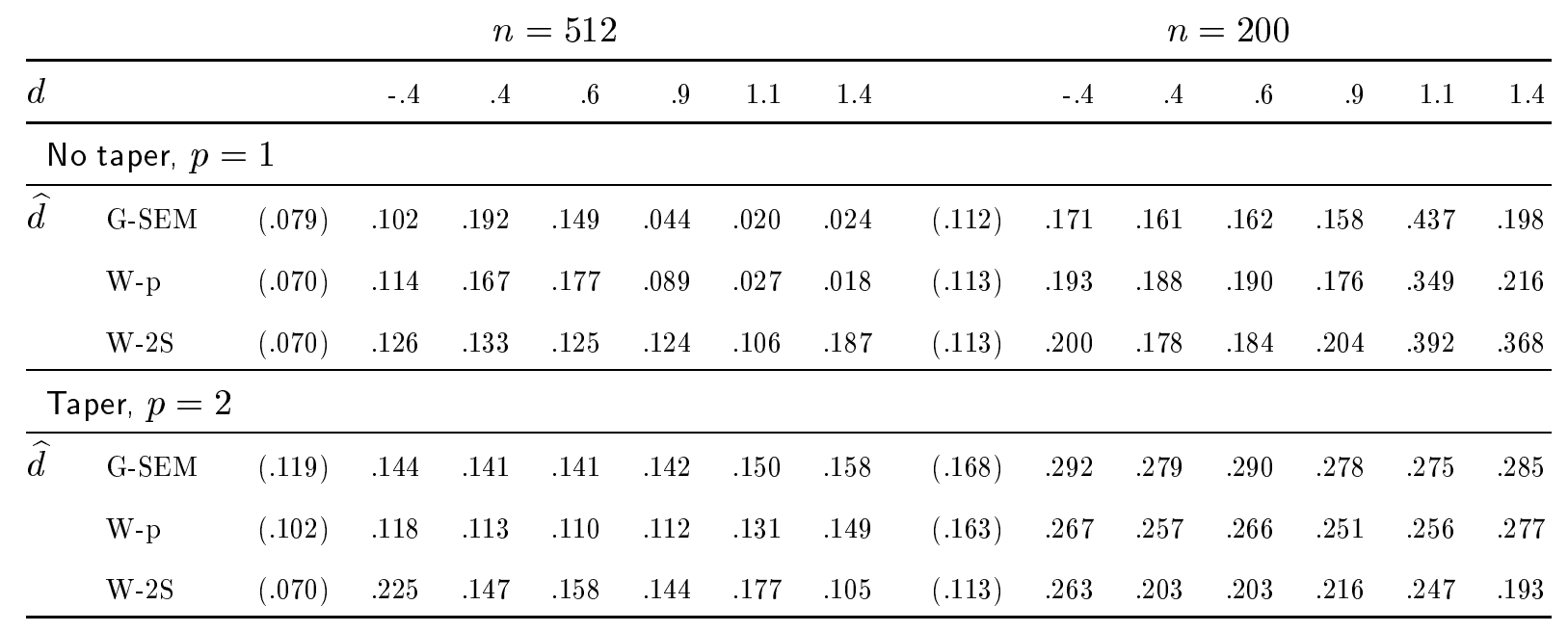

When no tapering is applied ( $p=1$ ) the estimation of $d$ breaks down if $d>1$, but even for these values of the memory parameter the two-step procedures give consistent estimates because the Whittle procedure tends to report $\widehat{d} \approx 1$, so the differenced series with these initial estimates of $d$ are stationary. The bias in Table 4 decreases for all estimates in sample size, and the large bias for semiparametric estimates can be explained in part by sub-optimal bandwidth choices. The asymptotic standard deviation gives a good indication of the variability of the Whittle estimates, but tends to increase slightly with the memory $d$ (see Table 5). The simulations confirm the consistency of Whittle tapered estimates for all $d$. Nevertheless the bias is larger than for two-step estimates and the standard deviations are also slightly larger than expected. This increment in variability of tapered Whittle leads to an increase in the variance of the twostep ML estimates, but not of the two-step Whittle estimates, so time domain estimation seems more sensitive to previous fractional differencing.

In Table 6 tapered Whittle estimation provides better results for the short memory ARMA parameters than for the memory parameter $d$, with very close behaviour to that of the two-step procedures in terms of bias, while in Table 7 the standard deviation, though larger, is very well approximated by the asymptotic outcome. However, for the first autoregressive parameter $\phi_{1}$, the tapered estimates produce larger biases than the other methods in some particular cases. Here the invariance of the results across $d$ is even more evident (except for $d>1$ and $p=1$ when untapered procedures yield inconsistent estimates). In conclusion, for the largest sample size the asymptotic theory gives a good approximation to the finite sample behaviour of Whittle estimates, confirming the uniform behaviour of the estimates across $d$, even for nonstationary series. 
Table 10: Bias of $\widehat{\theta}_{1}$ and $\widehat{\theta}_{2}$ for Bloomfield FEXP(2) Models

\begin{tabular}{|c|c|c|c|c|c|c|c|c|c|c|c|c|c|}
\hline \multicolumn{8}{|c|}{$n=512$} & \multicolumn{6}{|c|}{$n=200$} \\
\hline$d$ & & -.4 & .4 & .6 & .9 & 1.1 & 1.4 & -.4 & .4 & .6 & .9 & 1.1 & 1.4 \\
\hline \multicolumn{14}{|c|}{ No taper, $p=1$} \\
\hline \multirow[t]{2}{*}{$\widehat{\theta}_{1}$} & $\mathrm{~W}-\mathrm{p}$ & -.725 & -2.119 & -3.605 & -4.703 & -4.858 & -4.944 & -.752 & -1.074 & -1.365 & -2.364 & -1.813 & -3.001 \\
\hline & $\mathrm{W}-2 \mathrm{~S}$ & -.753 & -.823 & -.717 & -.864 & -1.580 & -3.648 & -.909 & -1.127 & -1.356 & -1.589 & -1.235 & -2.813 \\
\hline \multirow[t]{2}{*}{$\widehat{\theta}_{2}$} & $\mathrm{~W}-\mathrm{p}$ & .905 & 1.653 & 2.468 & 2.949 & 2.989 & 3.018 & 1.150 & 1.527 & 1.757 & 2.345 & 1.708 & 1.793 \\
\hline & $\mathrm{W}-2 \mathrm{~S}$ & .961 & .924 & .909 & 1.107 & 1.591 & 2.387 & 1.350 & 1.572 & 1.776 & 1.868 & 1.606 & 2.167 \\
\hline \multicolumn{14}{|c|}{ Taper, $p=2$} \\
\hline \multirow[t]{2}{*}{$\widehat{\widehat{\theta}_{1}}$} & $\mathrm{~W}-\mathrm{p}$ & .132 & .113 & .099 & .077 & -.007 & -.256 & .373 & .393 & .327 & .278 & .160 & -.304 \\
\hline & $\mathrm{W}-2 \mathrm{~S}$ & -3.580 & -1.031 & -1.155 & -1.302 & -1.691 & .618 & -3.711 & -1.479 & -1.435 & -2.024 & -2.457 & 2.008 \\
\hline \multirow[t]{2}{*}{$\widehat{\theta}_{2}$} & $\mathrm{~W}-\mathrm{p}$ & .040 & .032 & .029 & .044 & .209 & -.875 & .142 & .154 & .161 & .240 & .426 & -1.142 \\
\hline & $\mathrm{W}-2 \mathrm{~S}$ & 2.088 & .957 & 1.025 & 1.231 & 1.544 & 1.178 & 2.388 & 1.551 & 1.550 & 1.844 & 2.159 & 1.583 \\
\hline
\end{tabular}

\subsection{Exponential models}

In Tables 8 to 11 we report the results for exponential models with the same values of $d$ as used before. The conclusions for $n=200$ and for all untapered estimates of $d$ are rather negative, with large biases (Table 8) and variability (Table 9) relating to the asymptotic value, probably due to a difficult distinction between the short memory and long memory components of this particular model. Nevertheless, tapered Whittle estimation for $n=512$ produces for all $d$ reasonable biases and standard deviations, the smallest across all methods and quite close to the asymptotic ones, while both two-step estimations break down in many cases.

The superiority of tapered Whittle "W-p" estimates for the memory parameter of fractional exponential Bloomfield models carries over also for the short memory parameters $\theta_{1}$ and $\theta_{2}$, for which the untapered two-step procedures completely fail in capturing the true model for many parameter value combinations (Tables 10 and 11). Here Zhurbenko weights with $p=2$ for tapered estimates appear superior than the cosine bell in terms of bias for most values of $d$.

Our simulations agree with Dahlhaus' (1988) finding that tapering is desirable in estimating short memory parameters when the sd has peaks due to AR roots close to the unit circle, which are similar to the zero frequency singularity of the psd of fractionally integrated processes.

\section{ILLUSTRATIVE EXAMPLES}

In this section we analyze the two empirical series studied by Beran (1995), Chemical Process Temperature readings (Series C) and Chemical Process Concentration readings (Series A) from Box and Jenkins (1976), which are also among the series to which Robinson (1994b) applied his 
Table 11: Standard deviation of $\widehat{\theta}_{1}$ and $\widehat{\theta}_{2}$ for Bloomfield FEXP(2) Models

\begin{tabular}{|c|c|c|c|c|c|c|c|c|c|c|c|c|c|c|}
\hline \multicolumn{8}{|c|}{$n=512$} & \multicolumn{7}{|c|}{$n=200$} \\
\hline$d$ & & -.4 & .4 & .6 & .9 & 1.1 & 1.4 & & -.4 & .4 & .6 & .9 & 1.1 & 1.4 \\
\hline \multicolumn{15}{|c|}{ No taper, $p=1$} \\
\hline \multirow[t]{2}{*}{$\widehat{\theta}_{1}$} & W-p (.166) & .462 & .994 & 1.889 & .583 & .444 & .025 & $(.372)$ & .613 & .722 & .771 & .973 & 2.064 & 2.297 \\
\hline & $\mathrm{W}-2 \mathrm{~S}(.166)$ & .551 & .472 & .477 & .520 & .763 & 1.260 & $(.266)$ & .664 & .748 & .726 & .990 & .941 & 1.685 \\
\hline \multirow[t]{2}{*}{$\widehat{\theta}_{2}$} & W-p (.113) & .456 & .549 & .501 & .233 & .217 & .016 & $(.253)$ & .555 & .598 & .597 & .552 & 1.306 & 1.547 \\
\hline & $\mathrm{W}-2 \mathrm{~S}(.113)$ & .556 & .526 & .528 & .533 & .514 & .490 & $(.181)$ & .627 & .635 & .605 & .791 & .714 & .812 \\
\hline \multicolumn{15}{|c|}{ Taper, $p=2$} \\
\hline \multirow[t]{2}{*}{$\widehat{\theta}_{1}$} & $\mathrm{~W}-\mathrm{p}(.249)$ & .261 & .255 & .251 & .258 & .290 & .425 & $(.372)$ & .534 & .504 & .524 & .514 & .569 & .731 \\
\hline & $\mathrm{W}-2 \mathrm{~S}(.166)$ & 1.186 & .604 & .681 & .776 & .984 & .408 & $(.266)$ & 1.258 & 1.006 & 1.038 & 1.249 & 1.266 & .568 \\
\hline \multirow[t]{2}{*}{$\widehat{\theta}_{2}$} & W-p (.170) & .166 & .169 & .169 & .170 & .214 & .527 & $(.253)$ & .311 & .306 & .304 & .316 & .412 & .687 \\
\hline & $\mathrm{W}-2 \mathrm{~S}(.113)$ & .718 & .521 & .549 & .565 & .611 & .471 & $(.181)$ & .731 & .692 & .691 & .712 & .646 & .588 \\
\hline
\end{tabular}

Table 12: CHEMICAL SERIES-C. ARFIMA $(1, d, 0)$

\begin{tabular}{cccccccccc} 
& \multicolumn{1}{c}{$p=1$} & \multicolumn{3}{c}{ cos } \\
\hline & G-SEM & W-p & W-2S & G-SEM & W-p & W-2S & G-SEM & W-p & W-2S \\
\hline$\widehat{d}$ & .9928 & 1.0400 & .9788 & 1.4410 & .8676 & 1.0130 & 1.6370 & .9686 & .9930 \\
& $(.100)$ & $(.091)$ & - & $(.150)$ & $(.137)$ & - & $(.140)$ & $(.128)$ & - \\
\hline$\widehat{\phi}_{1}$ & - & .1157 & .8237 & - & .8389 & .7973 & - & .8263 & .8128 \\
& - & $(.116)$ & - & - & $(.141)$ & - & - & $(.143)$ & - \\
\hline$\widehat{\sigma}^{2}$ & - & .3171 & .0186 & - & .0162 & .0189 & - & .0150 & .0187 \\
\hline
\end{tabular}

score tests against fractional alternatives. We use the same estimates as in the simulations $(m=$ $25)$ and Zhurbenko's $(p=2)$ and cosine tapers. Both data tapers can deal with nonstationary series with $\mu=0$, but only tapering of order $p=2$ allows series with linear drift.

The conclusions are in line with Robinson's (1994b) and Beran's (1995) findings, and they contradict Box and Jenkins' (1976) finding of $\widehat{d}=1$ in series A and cast serious doubts about their $\widehat{d}=1$ in series $\mathrm{C}$, these values obtained by considering only integer degrees of differencing.

For series $\mathrm{C}$, all procedures in Table 12 for an $\operatorname{ARFIMA}(1, d, 0)$ model found values of $d$ indistinguishable from 1 (except both tapered Gaussian semiparametric estimates) and a highly significant first order autoregressive parameter of about 0.82 (in close agreement with Beran, 1995), which may explain why the tapered semiparametric estimates gave larger estimates of $d$ (clearly above 1) than the corresponding parametric methods. However, Whittle and semiparametric estimates without tapering $(p=1)$ may be inconsistent for this level of memory as is confirmed by the value of $\widehat{\sigma}^{2}$ (though the two-step estimate of $d$ is very close to the one with 
Table 13: CHEMICAL SERIES-C. Robinson's (1994) Tests of Nonstationarity

\begin{tabular}{|c|c|c|c|c|c|c|c|c|c|}
\hline$d_{o}$ & 0 & .25 & .5 & .75 & 1 & 1.25 & 1.5 & 1.75 & 2 \\
\hline$q=0$ & 28.3448 & 3.0751 & 29.4260 & 26.2129 & 20.0105 & 11.7572 & 4.5624 & -.0429 & -2.7152 \\
\hline$q=1$ & 13.1330 & 14.4261 & 12.6705 & 1.0989 & 6.5726 & 2.8689 & 1.3251 & -1.3023 & -2.8529 \\
\hline$q=2$ & 7.2360 & 8.0680 & 7.4209 & 5.7350 & 3.5236 & 1.2513 & -.6660 & -2.0282 & -2.9065 \\
\hline$q=3$ & 4.1008 & 4.8137 & 4.4391 & 3.3034 & 1.8890 & .3854 & -.9920 & -2.0581 & -2.7742 \\
\hline
\end{tabular}

original data).

We also estimated $\operatorname{FEXP}(q)$ models of orders $q=0,1,2$ and 3 (Table 14), the best fit produced by $q=2$. Estimates of $d$ decrease with the order $q$, from about $1.75(q=0)$ to 1.2 $(q=3)$. As for ARIMA models, Whittle estimates with the raw series are then likely to be inconsistent. We also used Robinson's (1994b) score test in Table 13 (using the same Bloomfield exponential models to explain high frequency behaviour), completing his results for an extended set of null values of $d$. The values reported are one-sided test statistics, with standard normal asymptotic distribution. The score tests always reject the hypothesis $d=2$, against $d<2$, and the hypothesis $d=1$, against $d>1$, but often do not reject $d=1.75,1.5$ and 1.25. The tests show a similar pattern to FEXP Whittle estimates, which contrasts with the ARIMA modelling in Table 12, but agrees with the semiparametric tapered estimates.

Table 14: CHEMICAL SERIES-C. Memory estimates $\widehat{d}, \operatorname{FEXP}(q)$

\begin{tabular}{lcccccc} 
& \multicolumn{2}{c}{$p=1$} & \multicolumn{3}{c}{ cos } \\
\hline$q=0$ & $\mathrm{~W}-\mathrm{p}$ & $\mathrm{W}-2 \mathrm{~S}$ & $\mathrm{~W}-\mathrm{p}$ & $\mathrm{W}-2 \mathrm{~S}$ & $\mathrm{~W}-\mathrm{p}$ & $\mathrm{W}-2 \mathrm{~S}$ \\
\hline$q=1$ & 1.1300 & 1.7522 & 1.7032 & 1.7509 & 1.7966 & 1.7510 \\
& $(.052)$ & $(.052)$ & $(.075)$ & $(.052)$ & $(.073)$ & $(.052)$ \\
\hline$q=2$ & 1.0688 & 1.5231 & 1.5110 & 1.6136 & 1.5685 & 1.6183 \\
& $(.083)$ & $(.083)$ & $(.120)$ & $(.083)$ & $(.116)$ & $(.083)$ \\
\hline$q=3$ & .9299 & 1.3703 & 1.2225 & 1.4082 & 1.2653 & 1.4125 \\
& $(.106)$ & $(.106)$ & $(.154)$ & $(.106)$ & $(.147)$ & $(.106)$ \\
\hline & .7529 & 1.2126 & 1.1313 & 1.3162 & 1.1964 & 1.3286 \\
& $(.125)$ & $(.125)$ & $(.181)$ & $(.125)$ & $(.174)$ & $(.125)$ \\
\hline
\end{tabular}

For series $\mathrm{A}$ the results were much more uniform. In this case the memory is noticeably smaller, about 0.45 as estimated for an $\operatorname{ARFIMA}(0, d, 1)$ model (Table 15) and now all estimates are expected to be consistent (Beran, 1995, reported $\widehat{d}=0.445)$. The tapered Whittle $(p=2)$ and the semiparametric (cosine bell) estimates reported slightly larger values than other procedures. Here the MA(1) $\psi_{1}$ parameter seems insignificant (except perhaps for the Whittle estimate with $p=2$, which is the method with a highest estimate of $d$ and largest trade-off between $d$ and the 
Table 15: CHEMICAL SERIES-A. ARFIMA $(0, d, 1)$

\begin{tabular}{cccccccccc} 
& \multicolumn{1}{c}{$p=1$} & \multicolumn{3}{c}{ cos } \\
\hline & G-SEM & W-p & W-2S & G-SEM & W-p & W-2S & G-SEM & W-p & W-2S \\
\hline$\widehat{d}$ & .4237 & .4408 & .4572 & .4674 & .5502 & .4592 & .5178 & .4515 & .4578 \\
& $(.100)$ & $(.067)$ & - & $(.150)$ & $(.104)$ & - & $(.140)$ & $(.096)$ & - \\
\hline$\widehat{\psi}_{1}$ & - & .0183 & .0570 & - & .1839 & .0500 & - & .1116 & .0577 \\
& - & $(.061)$ & - & - & $(.093)$ & - & - & $(.086)$ & - \\
\hline$\widehat{\sigma}^{2}$ & - & .0994 & .0972 & - & .0819 & .0974 & - & .0868 & .0971 \\
\hline
\end{tabular}

Table 16: CHEMICAL SERIES-A. Robinson's (1994) Tests of Nonstationarity

\begin{tabular}{lrrrrrrrrr}
$d_{o}$ & 0 & .25 & .5 & .75 & 1 & 1.25 & 1.5 & 1.75 & 2 \\
\hline$q=0$ & 16.0836 & 4.5696 & -1.5296 & -4.0191 & -5.1917 & -5.8752 & -6.3249 & -6.6391 & -6.8677 \\
$q=1$ & 8.0214 & 2.9196 & -.5299 & -2.5009 & -3.4693 & -4.0131 & -3.4103 & -4.0675 & -4.3663 \\
$q=2$ & 4.8408 & 1.7333 & -.9173 & -2.6523 & -3.6170 & -4.1686 & -4.5341 & -4.7653 & -4.7765 \\
$q=3$ & 2.8842 & 1.1621 & -.6467 & -2.0962 & -3.0060 & -3.5145 & -3.8202 & -3.9798 & -3.7932 \\
\hline
\end{tabular}

short memory part of the model). If we eliminate the parameter $\psi_{1}$ in a reduced $\operatorname{ARIMA}(0, d, 0)$ model (see Table 17), the estimates of $d$ now drop to about 0.41 , also with reduced standard deviations.

Table 17: CHEMICAL SERIES-A. ARFIMA $(0, d, 0)$

\begin{tabular}{lrrrrrr} 
& \multicolumn{2}{r}{$p=1$} & \multicolumn{2}{r}{$\cos$} \\
\hline & $\mathrm{W}-\mathrm{p}$ & $\mathrm{W}-2 \mathrm{~S}$ & $\mathrm{~W}-\mathrm{p}$ & $\mathrm{W}-2 \mathrm{~S}$ & $\mathrm{~W}-\mathrm{p}$ & $\mathrm{W}-2 \mathrm{~S}$ \\
\hline$\widehat{d}$ & .4286 & .4217 & .4171 & .4179 & .3692 & .4207 \\
& $(.056)$ & - & $(.083)$ & - & $.078)$ & - \\
\hline$\widehat{\sigma}^{2}$ & .0994 & .0973 & .0829 & .0983 & .0872 & .0973 \\
\hline
\end{tabular}

Robinson's (1994b) tests always reject $d=1$ and $d=0.75$ and find some evidence in support of $d=0.5$ and 0.25 for Series A (see Table 16), confirming the results for Whittle estimates of the FEXP model (Table 18), which, except in two cases, always give values between 0.34 and 0.47 .

\section{Appendix A: Technical assumptions and results}

In the following regularity conditions, statements concerning vector or matrix derivatives of $k(\lambda ; \theta)$ with respect to $\theta$ should be understood elementwise. They are similar to those in Conditions A of Fox and Taqqu (1986) or in Dahlhaus (1989) for parametric estimates or in Robinson 
Table 18: CHEMICAL SERIES-A. Memory estimates $\widehat{d}, \operatorname{FEXP}(q)$

\begin{tabular}{lcccccr} 
& \multicolumn{2}{c}{$p=1$} & & \multicolumn{2}{c}{ cos } \\
\hline$q=0$ & $\mathrm{~W}-\mathrm{p}$ & $\mathrm{W}-2 \mathrm{~S}$ & $\mathrm{~W}-\mathrm{p}$ & $\mathrm{W}-2 \mathrm{~S}$ & $\mathrm{~W}-\mathrm{p}$ & W-2S \\
\hline$q=1$ & .4286 & .4217 & .4171 & .4179 & .3692 & .4207 \\
& $(.056)$ & $(.056)$ & $(.081)$ & $(.056)$ & $(.078)$ & $(.056)$ \\
\hline$q=2$ & .4421 & .4571 & .5645 & .4554 & .4623 & .4582 \\
& $(.089)$ & $. .089)$ & $(.129)$ & $(.089)$ & $(.124)$ & $(.089)$ \\
\hline$q=3$ & .3771 & .4323 & .4198 & .4208 & .3206 & .4255 \\
& $(.113)$ & $(.113)$ & $(.165)$ & $(.114)$ & $(.158)$ & $(.113)$ \\
\hline & .3483 & .4470 & .4397 & .4469 & .2704 & .4301 \\
& $(.134)$ & $(.134)$ & $(.194)$ & $(.134)$ & $(.187)$ & $(.134)$ \\
\hline
\end{tabular}

(1995a, b) for semiparametric estimation of $d$, all holding for standard models such as fractional ARIMA's, fractional Gaussian noise or fractional exponential models (see e.g. Robinson, 1994a, and Beran, 1994). Denote $a_{\lambda}(\lambda ; \theta)=\frac{\partial}{\partial \lambda} a(\lambda ; \theta), a_{\lambda \theta}(\lambda ; \theta)=\frac{\partial}{\partial \lambda \partial \theta} a(\lambda ; \theta)$ and so on for any function $a$.

Assumption 1 (i) $\theta_{o}$ is an interior point of $\Theta$.

(ii) $k(\lambda ; \theta) \sim G_{\theta}|\lambda|^{-2 d}$ as $\lambda \rightarrow 0,0<G_{\theta}<\infty$, and is continuous and positive at all $\lambda \neq 0$ and $\theta \in \Theta$.

(iii) $\theta_{1} \neq \theta_{2}$ implies that $k\left(\lambda ; \theta_{1}\right) \neq k\left(\lambda ; \theta_{2}\right)$ on a set of positive Lebesgue measure.

Assumption $2 k(\lambda ; \theta)$ is differentiable in $\lambda$, with $k_{\lambda}(\lambda ; \theta)$ continuous at all $(\lambda, \theta), \lambda \neq 0$, and $k_{\lambda}(\lambda ; \theta)=O\left(|\lambda|^{-2 d-1}\right) \quad$ as $\lambda \rightarrow 0$.

Assumption 3 For each $\delta>0, k(\lambda ; \theta)$ is continuously differentiable in $\theta$ at all $(\lambda, \theta), \lambda \neq 0$, with $k_{\theta}^{-1}(\lambda ; \theta)=O\left(|\lambda|^{2 d-\delta}\right)$ as $\lambda \rightarrow 0$, and these derivatives are continuously differentiable in $\lambda$ at all $(\lambda, \theta), \lambda \neq 0$, with $k_{\lambda \theta}^{-1}(\lambda ; \theta)=O\left(|\lambda|^{2 d-1-\delta}\right)$ as $\lambda \rightarrow 0$.

The differentiability with respect to $\lambda$ is required to approximate discrete sums by integrals, even when $f(\lambda)$ has a singularity at the origin. To describe the stationary differenced series we introduce the following linear process assumption which is taken from Robinson (1995b), and is restrictive in the linearity it imposes, but not otherwise.

Assumption 4 We assume that

$$
U_{t}^{(s)}=\mu+\sum_{\ell=0}^{\infty} \alpha_{\ell} \epsilon_{t-\ell}, \quad \sum_{\ell=0}^{\infty} \alpha_{\ell}^{2}<\infty,
$$

with $\alpha_{\ell}=\alpha_{\ell}(\theta), \theta \in \Theta$, where $E\left[\epsilon_{t} \mid \mathcal{F}_{t-1}\right]=0, E\left[\epsilon_{t}^{2} \mid \mathcal{F}_{t-1}\right]=1$, a.s., $t=0, \pm 1, \ldots$, and $\mathcal{F}_{t}$ is the $\sigma$-field of events generated by $\epsilon_{t}, s \leq t$, and there exists a random variable $\epsilon$, such that $E \epsilon^{2}<\infty$ and for all $\eta>0$ and some $C>0, P\left(\left|\epsilon_{t}\right|>\eta\right) \leq C P(|\epsilon|>\eta)$. 
The following technical assumptions will be used to derive the asymptotic distribution of periodogram averages and parameter estimates. First we consider two smoothness conditions on $f$. When no taper is applied we will impose:

Assumption $5 \alpha(\lambda)=\sum_{\ell=0}^{\infty} \alpha_{\ell} e^{i \ell \lambda}$ is differentiable in $\lambda$ at all $(\lambda, \theta), \lambda \neq 0$ and $\frac{d}{d \lambda} \alpha(\lambda)=$ $O\left(|\alpha(\lambda)||\lambda|^{-1}\right)$ as $\lambda \rightarrow 0$.

Assumption 5 implies the differentiability of $f(\lambda)$ as stated in Assumption 2. This condition was imposed by Robinson (1995b) in a semiparametric context, with the observation that it applies to such parametric models as fractional ARIMAs. To use the tapering bias-reduction properties we assume stronger smoothness conditions.

Assumption $6 k_{\lambda}(\lambda ; \theta)$ is Lipschitz $(\beta-1)$ in $\lambda$, for some $1<\beta \leq 2$ and for all $(\lambda, \theta), \lambda \neq 0$ and for some $0<G_{\theta}, E_{\theta}<\infty, k(\lambda ; \theta)=G_{\theta}|\lambda|^{-2 d}+E_{\theta}|\lambda|^{-2 d+\beta}+o\left(|\lambda|^{-2 d+\beta}\right)$ as $\lambda \rightarrow 0$.

In particular, with $\beta>1$, Assumption 6 implies that, for $|\lambda|<\lambda_{j} / 2,0<j<n / 2$,

$$
f\left(\lambda_{j}-\lambda\right)=f\left(\lambda_{j}\right)-\lambda \frac{d}{d \lambda} f\left(\lambda_{j}\right)+O\left(\lambda_{j}^{-\beta-2 d}|\lambda|^{\beta}\right)
$$

as $\lambda \rightarrow 0$, which is the basis for the tapering bias reduction. For the asymptotic distribution of the estimates and related quadratic forms we need also an extra condition about the fourth moments of the linear innovations, again taken from Robinson (1995b), and two additional conditions to approximate the asymptotic covariance matrix of $\widehat{\theta}$.

Assumption 7 Assumption 4 holds and for finite constants $\mu_{3}$ and $\mu_{4}, E\left[\epsilon_{t}^{3} \mid \mathcal{F}_{t-1}\right]=\mu_{3}$, $E\left[\epsilon_{t}^{4} \mid \mathcal{F}_{t-1}\right]=\mu_{4}$, a.s., $t=0, \pm 1, \ldots$

Assumption $8 k(\lambda ; \theta)$ has two continuous derivatives in $\theta$ at all $(\lambda, \theta), \lambda \neq 0$, with $k_{\theta \theta^{\prime}}^{-1}(\lambda ; \theta)=$ $O\left(|\lambda|^{2 d-\delta}\right)$ as $\lambda \rightarrow 0$ for each $\delta>0$, and these derivatives are continuously differentiable in $\lambda$ at all $(\lambda, \theta), \lambda \neq 0$, with $k_{\lambda \theta \theta^{\prime}}^{-1}(\lambda ; \theta)=O\left(|\lambda|^{2 d-1-\delta}\right)$ as $\lambda \rightarrow 0$.

Assumption $9 \int_{-\pi}^{\pi}\left\{k^{-1}(\lambda ; \theta)+\log k(\lambda ; \theta)\right\} d \lambda$ can be continuously differentiated twice (with respect to $\theta)$ under the integral sign and $\Sigma_{o}^{-1}$ exists.

We review now some results obtained by Robinson (1995a) and Velasco (1999a) for the (tapered) DFT of possibly nonstationary time series. The following conditions on $f_{U^{(s)}}$, which hold under Assumptions 1 and 2, were assumed in these papers.

Assumption 10 For some $0<G<\infty, d>-\frac{1}{2}, s=\left\lfloor d+\frac{1}{2}\right\rfloor, f_{U^{(s)}}(\lambda)=G|\lambda|^{-2(d-s)}+$ $o\left(|\lambda|^{-2(d-s)}\right)$ as $\lambda \rightarrow 0$. 
Assumption $11 f_{U^{(s)}}(\lambda)$ has bounded derivative at all $\lambda \neq 0$, and $d>-\frac{1}{2}, s=\left\lfloor d+\frac{1}{2}\right\rfloor$, $\frac{d}{d \lambda} f_{U^{(s)}}(\lambda)=O\left(|\lambda|^{-2(d-s)-1}\right)$ as $\lambda \rightarrow 0$.

First we analyze the covariance matrix of the raw DFT $w\left(\lambda_{j}\right)$, for frequencies $\lambda_{j} \rightarrow 0$ and $\lambda_{j} \rightarrow \nu \in(0, \pi]$ as $n \rightarrow \infty$. Define $v(\lambda)=w(\lambda) / f(\lambda)^{1 / 2}$.

Theorem $3[p=1]$ Under Assumptions 10 and 11, $d \in\left(-\frac{1}{2}, 1\right)\left(\mu=0\right.$ if $\left.d \geq \frac{1}{2}\right)$, for any sequences of positive integers $j=j(n)$ and $k=k(n)$ such that $1 \leq k<j \leq n / 2$, defining $\gamma_{j, k} \equiv(j k)^{d-1} \log (k+1)$, as $n \rightarrow \infty$,

(a) $E\left[v\left(\lambda_{j}\right) \bar{v}\left(\lambda_{j}\right)\right]=1+O\left(j^{-1} \log (j+1)+\gamma_{j, j}\right)$,

(b) $E\left[v\left(\lambda_{j}\right) v\left(\lambda_{j}\right)\right]=O\left(j^{-1} \log (j+1)+\gamma_{j, j}\right)$,

(c) $E\left[v\left(\lambda_{j}\right) \bar{v}\left(\lambda_{k}\right)\right], E\left[v\left(\lambda_{j}\right) v\left(\lambda_{k}\right)\right]=O\left(k^{-1} \log j+\gamma_{j, k}\right)$.

For values $d \geq 1$ the periodogram is not asymptotically unbiased for $f$ as $j$ increases. Tapering allows a reduction in the order of magnitude of the bounds in Theorem 3, making possible the approximation of psd with larger $d$. Thus, with the cosine bell taper similar results go through for any $d<\frac{3}{2}$. Other tapers reduce even more the bias and allow consideration of values $d \geq \frac{3}{2}$. However, the full advantage of the tapers only shows up when we assume further smoothness conditions on $f$ :

Assumption $12 f(\lambda)$ satisfies a Lipschitz condition of degree $\beta \leq 1$ for all $\lambda \neq 0$, or $f(\lambda)$ is differentiable and $\frac{d}{d \lambda} f(\lambda)$ satisfies a Lipschitz condition of degree $\beta \in(1,2]$ for all $\lambda \neq 0$, and for some $0<G, E_{\beta}<\infty, d>-\frac{1}{2}, s=\left\lfloor d+\frac{1}{2}\right\rfloor$, as $\lambda \rightarrow 0, f_{U^{(s)}}(\lambda)=G|\lambda|^{-2(d-s)}+E_{\beta}|\lambda|^{-2(d-s)+\beta}+$ $o\left(|\lambda|^{-2(d-s)+\beta}\right)$.

This condition holds under Assumption 6 for $\beta>1$ (see also (10)). We consider now the full cosine bell taper (4) and define the normalized cosine-tapered DFT $v_{\cos }(\lambda)=w(\lambda) / f(\lambda)^{1 / 2}$.

Theorem 4 [Cosine bell] Under Assumptions 11 and 12, $d \in\left(-\frac{1}{2}, \frac{3}{2}\right)$ (and $\mu=0$ if $d \geq \frac{1}{2}$ ), for any sequences of positive integers $j=j(n)$ and $k=k(n), 3<k+2<j \leq n / 2$, defining $\gamma_{j, k} \equiv(j k)^{d-3} \log (k+1)$, as $n \rightarrow \infty$,

(a) $\left.E\left[v_{\cos } \lambda_{j}\right) \overline{v_{\cos }}\left(\lambda_{j}\right)\right]=1+O\left(\min \left\{j^{-\beta}, j^{-1}\right\}+\gamma_{j, j}\right)$,

(b) $E\left[v_{\cos }\left(\lambda_{j}\right) v_{\cos }\left(\lambda_{j}\right)\right]=O\left(j^{-4}+\gamma_{j, j}\right)$,

(c) $E\left[v_{\cos }\left(\lambda_{j}\right) \overline{v_{\cos }}\left(\lambda_{k}\right)\right], E\left[v_{\cos }\left(\lambda_{j}\right) v_{\cos }\left(\lambda_{k}\right)\right]=O\left(k^{-1}|j-k|^{-2}+\gamma_{j, k}\right)$,

and when $k=j+1$ and $k=j+2$ all the previous statements are true with

(c') $E\left[v_{\cos }\left(\lambda_{j}\right) \overline{v_{\cos }}\left(\lambda_{j+1}\right)\right]=-\frac{2}{3}+O\left(j^{-1}+\gamma_{j, j}\right)$,

(c") $E\left[v_{\cos }\left(\lambda_{j}\right) \overline{v_{\cos }}\left(\lambda_{j+2}\right)\right]=\frac{1}{6}+O\left(j^{-1}+\gamma_{j, j}\right)$.

We now analyze the covariance matrix of the (normalized) tapered DFT with tapers of order $p>1, v_{p}(\lambda)$. The periodogram is now asymptotically unbiased for any $p>d$ at frequencies 
$\lambda_{j p}, j$ integer, but tapering destroys the orthogonality of the sine and cosine transforms at close frequencies.

Theorem $5[p \geq 2]$ Under Assumptions 11 and 12, $d>-\frac{1}{2}$, for $f_{U^{(s)}}$, a data taper of order $p=2,3, \ldots$, with $p>d[p \geq s+1$ if $\mu \neq 0]$, for any sequences of positive integers $k=k(n)$ and $j=j(n), 1 \leq k<j \leq n /(2 p)$, defining $\gamma_{j, k} \equiv(j k)^{d-p} \log (k+1)$, as $n \rightarrow \infty$,

(a) $E\left[v_{p}\left(\lambda_{j p}\right) \overline{v_{p}}\left(\lambda_{j p}\right)\right]=1+O\left(\min \left\{j^{-\beta}, j^{-1}\right\}+\gamma_{j, j}\right)$,

(b) $E\left[v_{p}\left(\lambda_{j p}\right) v_{p}\left(\lambda_{j p}\right)\right]=O\left(j^{-p}+j^{-1-p} \log (j+1)+\gamma_{j, j}\right)$,

(c) $E\left[v_{p}\left(\lambda_{j p}\right) \overline{v_{p}}\left(\lambda_{k p}\right)\right], E\left[v_{p}\left(\lambda_{j p}\right) v_{p}\left(\lambda_{k p}\right)\right]=O\left(k^{-1}|j-k|^{1-p}+k^{-1}|j-k|^{-p} \log n+|j-k|^{-p}+\right.$ $\left.\gamma_{j, k}\right)$.

In (c) the term $\log n$ only appears if $p=2$. Theorem 4's bounds are similar to Theorem 5's for $p=3$, at all Fourier frequencies but only for $d<\frac{3}{2}$, so the cosine bell taper shares some properties with tapers of order $p=3$, though it cannot filter out polynomial trends.

We now present two lemmas for the consistency and uniform consistency in probability of discrete averages of periodogram ordinates of possibly nonstationary (and tapered) observations, which can be seen as specific quadratic forms of $X_{t}, t=1, \ldots, n$. All functions are assumed to be periodic of period $2 \pi$. Proofs are collected in Appendix B.

Lemma 6 For an even function $\psi(\lambda)$, differentiable at all $\lambda \neq 0$, let $\psi(\lambda)=O\left(f^{-1}(\lambda)|\lambda|^{-\delta}\right)$ and $\frac{d}{d \lambda} \psi(\lambda)=O\left(f^{-1}(\lambda)|\lambda|^{-1-\delta}\right)$ as $\lambda \rightarrow 0, \delta \in(0,1)$, let $H=\int_{-\pi}^{\pi} \psi(\lambda) f(\lambda) d \lambda<\infty$, and $H_{n}=$ $(2 \pi p / n) \sum_{j(p)} \psi\left(\lambda_{j}\right) I^{p}\left(\lambda_{j}\right)$, for $p=1,2, \ldots$ Then, under Assumptions 1, 2 and $4, H_{n} \rightarrow_{p} H$ as $n \rightarrow \infty$ if $p \geq\left\lfloor d_{o}+\frac{1}{2}\right\rfloor+1$, [only $p>d_{o}$ if $\mu=0$ or $\left.d_{o}<\frac{1}{2}\right]$.

Lemma 7 For an even function $\psi(\lambda ; \theta)$, let $\psi(\lambda ; \theta)=O\left(f^{-1}(\lambda)|\lambda|^{-\delta}\right)$ as $\lambda \rightarrow 0$ be continuously differentiable in $\lambda$ and $\theta$ at all $(\lambda, \theta), \lambda \neq 0, \theta \in \Theta_{1}$ compact, with $\psi_{\lambda}(\lambda ; \theta)=$ $O\left(f^{-1}(\lambda)|\lambda|^{-1-\delta}\right)$ and $\psi_{\theta}(\lambda ; \theta)=O\left(f^{-1}(\lambda)|\lambda|^{-\delta}\right), \delta=\delta(\theta) \in(0,1)$ for all $\theta \in \Theta_{1}$, and let $H(\theta)=\int_{-\pi}^{\pi} \psi(\lambda ; \theta) f(\lambda) d \lambda<\infty$, and, for $p=1,2, \ldots, H_{n}(\theta)=(2 \pi p / n) \sum_{j(p)} \psi\left(\lambda_{j} ; \theta\right) I^{p}\left(\lambda_{j}\right)$. Then, under Assumptions 1, 2 and 4, $\sup _{\theta \in \Theta_{1}}\left|H_{n}(\theta)-H(\theta)\right| \rightarrow_{p} 0$ as $n \rightarrow \infty$ if $p \geq\left\lfloor d_{o}+\frac{1}{2}\right\rfloor+1$, [only $p>d_{o}$ if $\mu=0$ or $d_{o}<\frac{1}{2}$ ].

The condition on $\delta(\theta)$ in Lemma 2 restricts the permitted values of $\theta$ in the compact set $\Theta_{1}$. The next lemma analyzes the asymptotic distribution of the periodogram averages.

Lemma 8 In addition to the Assumptions of Lemma 1 on $\psi$, where now $\delta>0$ is arbitrarily small, under Assumptions 1, 2, 4 and 7, assumptions (i) and (ii) of Theorem 2 and $H=0$, as $n \rightarrow \infty, \sqrt{n} H_{n} \rightarrow_{d} N\left(0,4 \pi p \Phi_{p} \int_{-\pi}^{\pi} \psi(\lambda) \psi^{\prime}(\lambda) f^{2}(\lambda) d \lambda\right)$, where $\psi^{\prime}(\lambda)$ stands for the transpose of $\psi(\lambda)$.

Lemma 9 Under the conditions of Theorem 1, for $0<\varepsilon<\frac{1}{2},(2 \pi p / n) \sum_{j(p)}\left\{I^{p}\left(\lambda_{j}\right)-f\left(\lambda_{j}\right)\right\}$ $\times\left|\lambda_{j}\right|^{2\left(d_{o}+\varepsilon-1 / 2\right)} \rightarrow_{p} 0$ as $n \rightarrow \infty$. 
Lemma 10 For a function $g$, even and periodic (of period $2 \pi$ ), satisfying $g(\lambda)=O\left(|\lambda|^{-\delta}\right.$ ) as $\lambda \rightarrow 0,0<\delta<1$, and a Lipschitz condition of degree $\alpha \in(0,1]$ with constant $O\left(|\lambda|^{-1-\delta}\right)$, i.e., for $\omega>0,|g(\lambda+\omega)-g(\lambda)|=O\left(|\lambda|^{-1-\delta} \omega^{\alpha}\right) \quad$ as $\lambda \rightarrow 0, p=1,2, \ldots$, then as $n \rightarrow \infty$, $\int_{0}^{2 \pi} g(\lambda) d \lambda-(2 \pi p / n) \sum_{j(p)} g\left(\lambda_{j}\right)=O\left(n^{\delta-\alpha}\right)$.

Lemma 11 Under the Assumptions of Lemma 3, for $g=\psi f, \lim _{n \rightarrow \infty} V_{n}=\Phi_{p} 4 \pi \int_{-\pi}^{\pi} g^{2}(\lambda) d \lambda$ where

$$
\begin{aligned}
V_{n}= & 4 \sum_{t=1}^{n-1} h_{t}^{2} \sum_{s=1}^{n-t} h_{s+t}^{2}(4 \pi)^{2}\left(\sum_{1}^{n} h_{t}^{2}\right)^{-2} \\
& \times \frac{p}{n} \sum_{j=1}^{n^{*}} \sum_{k=1}^{n^{*}} g\left(\lambda_{j p}\right) \cos s \lambda_{j p} g\left(\lambda_{k p}\right) \cos s \lambda_{k p} .
\end{aligned}
$$

Lemma 12 Under Assumptions 1 through 4, 8 and 9, with $p \geq\left\lfloor d_{o}+\frac{1}{2}\right\rfloor+1$ [only $p>d_{o}$ if $\mu=0$ or $\left.d_{o}<\frac{1}{2}\right]$, as $n \rightarrow \infty, \widehat{\theta}=\theta_{o}+(2 \pi p / n) \sum_{j(p)} \rho_{o}\left(\lambda_{j}\right)\left[I^{p}\left(\lambda_{j}\right)-f\left(\lambda_{j}\right)\right]+o_{p}\left(n^{-1 / 2}\right)$, where $\rho_{o}(\lambda)=\Xi_{o}^{-1} k_{\theta}^{-1}\left(\lambda ; \theta_{o}\right)$, and $\Xi_{o}=\sigma_{o}^{2} \Sigma_{o} /(2 \pi)$.

Lemma 13 If the sequence $\left\{h_{t}\right\}$ is a data taper of order $p$, for $0<j<n / 2$, as $n \rightarrow \infty, h\left(\lambda_{j}\right)=$ $O\left(j^{-p}\right)$.

Lemma 14 If the sequence $\left\{h_{t}\right\}$ is a data taper of order $p$, for $0<j<n$, as $n \rightarrow \infty$, $\sum_{t=1}^{n-1} h_{t}^{2} \sum_{s=1}^{n-t} h_{s+t}^{2} \cos s \lambda_{j}=\frac{1}{2}\left(\sum_{t=1}^{n} h_{t}^{2} \cos t \lambda_{j}\right)^{2}+O(n)$.

\section{Appendix B: Proofs}

Proof of Theorem 1. We follow the proof in two steps of Theorem 1 in Robinson (1995b).

First step. Denote $\theta^{(1)}=d$ and $\Theta_{1}=\left\{d: \nabla_{1} \leq d \leq \nabla_{2}\right\} \times \Theta^{(-1)}$, if $\nabla_{1}>d_{o}-\frac{1}{2}$, or otherwise $\Theta_{1}=\left\{d: d_{o}-\frac{1}{2}+\varepsilon \leq d \leq \nabla_{2}\right\} \times \Theta^{(-1)}$, for some $0<\varepsilon<\frac{1}{2}$. Define $\widehat{\theta}_{1}=\arg \min _{\theta \in \Theta_{1}} Q_{n}(\theta)$ and $Q(\theta)=\int_{-\pi}^{\pi} f(\lambda) k^{-1}(\lambda ; \theta) d \lambda$.

$\widehat{\theta}_{1} \rightarrow{ }_{p} \theta_{o}$ follows by a standard argument for consistency of implicitly-defined extremum estimates if we can write $Q_{n}(\theta)-Q_{n}\left(\theta_{0}\right)=S(\theta)-U(\theta)$, where $S(\theta)$ is nonstochastic and constant over $n$, such that for all $\varepsilon>0$ there exists $\eta>0$ such that $\inf _{\left\|\theta-\theta_{o}\right\| \geq \varepsilon} S(\theta) \geq \eta$, and also $\sup _{\theta \in \Theta_{1}}|U(\theta)| \rightarrow_{p}$ 0. Since there is a unique minimum of $Q(\theta)$ at $\theta=\theta_{o}$ from the identifiability conditions in Assumption 1, setting $S(\theta)=Q(\theta)-Q\left(\theta_{o}\right)$ the condition on $S$ follows from the uniform continuity of $Q(\theta)$ on $\Theta_{1}$. The condition on $U(\theta)=Q_{n}(\theta)-Q_{n}\left(\theta_{o}\right)-Q(\theta)+Q\left(\theta_{o}\right)$ follows because $\sup _{\theta \in \Theta_{1}}\left|Q_{n}(\theta)-Q(\theta)\right| \rightarrow_{p} 0$ using Lemmas $2\left[\psi(\lambda ; \theta)=k^{-1}(\lambda ; \theta)\right]$ and $5[g(\lambda)=$ $\psi(\lambda ; \theta) f(\lambda)]$ to approximate uniformly in $\Theta_{1}$ integrals with sums, and using this last lemma we get that $\sup _{\theta \in \Theta_{1}}\left|Q_{n}\left(\theta_{o}\right)-Q\left(\theta_{o}\right)\right| \rightarrow 0$ as $n \rightarrow \infty$.

Second step. Recall that $\Theta_{1}=\left\{d: \nabla \leq d \leq \nabla_{2}\right\} \times \Theta^{(-1)}$, where $\nabla=\nabla_{1}$ when $d_{o}<\frac{1}{2}+\nabla_{1}$ and $d_{o}-\frac{1}{2}<\nabla \leq d_{o}$ otherwise. When $d_{o} \geq \frac{1}{2}+\nabla_{1}$, define $\Theta_{2}=\left\{d: \nabla_{1} \leq d<\nabla\right\} \times \Theta^{(-1)}$, or 
setting $\varepsilon=\nabla-\left(d_{o}-\frac{1}{2}\right)$ with $\varepsilon=\varepsilon\left(\theta_{o}\right)>0$, (to be chosen later, in $\left(0, \frac{1}{2}\right), \Theta_{2}=\left\{d: \nabla_{1} \leq d<\right.$ $\left.d_{o}-\frac{1}{2}+\varepsilon\right\} \times \Theta^{(-1)}$.

Then $\widehat{\theta}_{1} \rightarrow_{p} \theta_{o}$ if $\Theta_{1}=\Theta$ and the theorem is proved. Consider now the situation where $\Theta_{2}$ is not empty. We want to show that $\widehat{\theta}-\widehat{\theta}_{1} \rightarrow_{p} 0$. For any $\delta>0$

$$
\begin{aligned}
P\left(\left\|\widehat{\theta}-\widehat{\theta}_{1}\right\| \geq \delta\right) & \leq P\left(\inf _{\theta \in \Theta_{2}} Q_{n}(\theta) \leq \min _{\theta \in \Theta_{1}} Q_{n}(\theta)\right) \\
& \leq P\left(\inf _{\theta \in \Theta_{2}} Q_{n}(\theta)-Q\left(\theta_{o}\right) \leq \delta^{\prime}\right) \\
& +P\left(\left|Q_{n}\left(\widehat{\theta}_{1}\right)-Q\left(\theta_{o}\right)\right| \geq \delta^{\prime}\right)
\end{aligned}
$$

for any $\delta^{\prime}>0$. Since $\widehat{\theta}_{1} \rightarrow_{p} \theta_{o}$ (for any $\varepsilon>0$ in the definition of $\Theta_{1}$ ) the second probability tends to zero as $n \rightarrow \infty$, for any $\delta^{\prime}>0$. Write $k^{*}(\lambda ; \theta)=|\lambda|^{2 \theta^{(1)}} k(\lambda ; \theta)$ so $f\left(\lambda ; \sigma^{2}, \theta\right)=$ $\frac{\sigma^{2}}{2 \pi}|\lambda|^{-2 \theta^{(1)}} k^{*}(\lambda ; \theta), 0<c_{1}<k^{*}(\lambda ; \theta)<c_{2}<\infty$, say, for all $\lambda, \theta$, under Assumption 1 . To show that the first probability in (11) is negligible, note that

$$
\begin{aligned}
\inf _{\theta \in \Theta_{2}} Q_{n}(\theta) & =\inf _{\theta \in \Theta_{2}} \frac{2 \pi p}{n} \sum_{j(p)} I^{p}\left(\lambda_{j}\right) k^{-1}\left(\lambda_{j} ; \theta\right) \\
& \geq \frac{2 \pi p}{n c_{2}} \sum_{j(p)} I^{p}\left(\lambda_{j}\right)\left|\lambda_{j}\right|^{2\left(d_{o}+\varepsilon-1 / 2\right)} .
\end{aligned}
$$

The last sum converges in probability (see Lemmas 4 and 5 again) to

$$
\begin{aligned}
& \frac{2 \pi p}{n c_{2}} \sum_{j(p)} f\left(\lambda_{j}\right)\left|\lambda_{j}\right|^{2\left(d_{o}+\varepsilon-1 / 2\right)}=\frac{\sigma^{2}}{2 \pi} \frac{2 \pi p}{n c_{2}} \sum_{j(p)} k^{*}\left(\lambda_{j}\right)\left|\lambda_{j}\right|^{2 \varepsilon-1} \\
& \sim \frac{\sigma^{2}}{2 \pi c_{2}} \int_{-\pi}^{\pi} k^{*}(\lambda)|\lambda|^{2 \varepsilon-1} d \lambda \\
& \geq \frac{\sigma^{2}}{2 \pi} \frac{c_{1}}{c_{2}} \int_{-\pi}^{\pi}|\lambda|^{2 \varepsilon-1} d \lambda=\frac{\sigma^{2}}{2 \pi} \frac{c_{1}}{c_{2}} \frac{\pi^{2 \varepsilon}}{\varepsilon}=C(\varepsilon)>0, \quad \text { say }
\end{aligned}
$$

and $C(\varepsilon)$ can be made as large as desired for any $f$ and $\theta_{o}$, by choice of $\varepsilon$. Fix $\delta^{\prime}>0$ and then pick $\varepsilon>0$ such that $C(\varepsilon)>Q\left(\theta_{o}\right)+2 \delta^{\prime}$, define $\Theta_{1}$ and $\widehat{\theta}_{1} \rightarrow_{p} \theta_{o}$ so the first term in (11) tends to zero as $n \rightarrow \infty$, and thus $\widehat{\theta} \rightarrow_{p} \widehat{\theta}_{1}$. The consistency of $\widehat{\sigma}^{2}$ follows from that of $\widehat{\theta}$ and Lemma 2 .

Proof of Theorem 2. Define $\psi_{o}(\lambda)=k_{\theta}^{-1}\left(\lambda ; \theta_{o}\right)$, and then use Theorem 1 and Lemmas 3 and 7 and that $\Sigma_{o}^{-1} \int_{-\pi}^{\pi} \psi_{o}(\lambda) \psi_{o}^{\prime}(\lambda) k^{2}\left(\lambda ; \theta_{o}\right) d \lambda \Sigma_{o}^{-1}=\Sigma_{o}^{-1}$.

Proof of Lemma 1. We prove the lemma by approximating the periodogram of the (possibly tapered) observed series by that of the (possibly tapered) linear innovations, $I^{p, \epsilon}\left(\lambda_{j}\right)$, times the transfer function, including the unit root filters. Define, $p=1,2, \ldots, H_{n}^{\epsilon}=(2 \pi)^{2}(p / n)$ $\times \sum_{j(p)} \psi\left(\lambda_{j}\right) f\left(\lambda_{j}\right) I^{p, \epsilon}\left(\lambda_{j}\right)$, so using Theorems 3 and 5 in Appendix A, and evenness of all functions,

$$
H_{n}-H_{n}^{\epsilon}=\frac{4 \pi}{n} \sum_{j=1}^{n^{\star}} \psi\left(\lambda_{j p}\right)\left[I^{p}\left(\lambda_{j p}\right)-2 \pi f\left(\lambda_{j p}\right) I^{p, \epsilon}\left(\lambda_{j p}\right)\right]+o_{p}(1),
$$


where $n^{\star}=\lfloor[(n / p)-1] / 2\rfloor$. We now distinguish the cases with and without tapering.

No tapering $[p=1]$. Consider the case with $d_{o}<1, \mu=0$, or $d_{o}<\frac{1}{2}$, for any $\mu$, and $s=1$ or 0 . Using the same arguments to those used in the proof of Theorem 2 of Velasco (1999b) (see also the proof of Theorem 1 of Robinson, 1995b), $0<j \leq n / 2, d_{o} \in\left(-\frac{1}{2}, 1\right), f\left(\lambda_{j}\right)=$ $(2 \pi)^{-1}\left|1-e^{-i \lambda_{j}}\right|^{-2 s}\left|\alpha\left(\lambda_{j}\right)\right|^{2}$, to show that $E\left|I\left(\lambda_{j}\right)-2 \pi f\left(\lambda_{j}\right) I^{\epsilon}\left(\lambda_{j}\right)\right|=O\left(f\left(\lambda_{j}\right)\left[j^{-1 / 2}(\log j)^{1 / 2}+\right.\right.$ $\left.\left.j^{d_{o}-1}(\log j)^{1 / 2}\right]\right)$, we find from (12) that, using $\psi\left(\lambda_{j}\right)=O\left(f^{-1}\left(\lambda_{j}\right)\left|\lambda_{j}\right|^{-\delta}\right), 0<\delta<1$,

$$
\begin{aligned}
H_{n}-H_{n}^{\epsilon} & =O_{p}\left(n^{-1 / 2}(\log n)^{1 / 2}+n^{\delta-1}(\log n)^{3 / 2}\right) \\
& +O_{p}\left(n^{d_{o}-1}(\log n)^{1 / 2}\right)+o_{p}(1)
\end{aligned}
$$

which is $o_{p}(1)$ if $d_{o}<1$. The expectation of $H_{n}^{\epsilon}$ is with Lemma 5 , using the continuity of $f(\lambda)$ and $\psi(\lambda)$, and the integrability of $f(\lambda) \psi(\lambda), E\left[H_{n}^{\epsilon}\right]=(2 \pi / n) \sum_{j=1}^{n-1} \psi\left(\lambda_{j}\right) f\left(\lambda_{j}\right) \sim \int_{-\pi}^{\pi} \psi(\lambda) f(\lambda) d \lambda<$ $\infty$, as $n \rightarrow \infty$. Now, by summation by parts, for a positive constant $C$,

$$
\begin{aligned}
& \left|\frac{4 \pi}{n} \sum_{1}^{n^{\star}} f\left(\lambda_{j}\right) \psi\left(\lambda_{j}\right)\left\{2 \pi I^{\epsilon}\left(\lambda_{j}\right)-1\right\}\right| \\
& \leq \frac{C}{n} \sum_{r=1}^{n^{\star}}\left|f\left(\lambda_{r}\right) \psi\left(\lambda_{r}\right)-f\left(\lambda_{r+1}\right) \psi\left(\lambda_{r+1}\right)\right|\left|\sum_{j=1}^{r}\left\{2 \pi I^{\epsilon}\left(\lambda_{j}\right)-1\right\}\right| \\
& +\frac{C}{n}\left|\sum_{1}^{n^{\star}}\left\{2 \pi I^{\epsilon}\left(\lambda_{j}\right)-1\right\}\right|\left|f\left(\lambda_{n / 2}\right) \psi\left(\lambda_{n / 2}\right)\right|
\end{aligned}
$$

Following the discussion in Robinson (1995b, pp. 1637-8), we obtain that, for $1 \leq r \leq n / 2$,

$$
\left|\sum_{1}^{r}\left\{2 \pi I^{\epsilon}\left(\lambda_{j}\right)-1\right\}\right|=o_{p}(r)+O_{p}\left(r^{1 / 2}\right),
$$

and using the properties of $f(\lambda)$ and $\psi(\lambda)$ and the mean value theorem, (13) is $o_{p}\left(n^{-1} \sum_{r=1}^{n} \lambda_{r}^{-1-\delta} n^{-1} r+1\right)=o_{p}(1)$.

Tapering $[p>1]$. We obtained in the proof of Theorem 5 in Velasco (1999b), that under the conditions of this lemma, $E\left|I^{p}\left(\lambda_{j p}\right)-2 \pi I^{p, \epsilon}\left(\lambda_{j p}\right)\right|=O\left(f\left(\lambda_{j p}\right)\left[j^{-1 / 2}+j^{d_{o}-p}(\log j)^{1 / 2}\right]\right), 0<j<$ $n /(2 p)$, so $H_{n}-H_{n}^{\epsilon}=O_{p}\left(n^{\delta-1} \log n+n^{-1 / 2}+n^{d_{o}-p}(\log n)^{1 / 2}\right)$, which is $o_{p}(1)$ if $p>d_{o}$. The expectation of $H_{n}^{\epsilon}$ for $p>1$ is calculated as for $p=1$. Now we can write

$$
\begin{aligned}
& 2 \pi I^{p, \epsilon}\left(\lambda_{j p}\right)-1=\frac{1}{\sum h_{t}^{2}} \sum_{t=1}^{n} h_{t}^{2}\left(\epsilon_{t}^{2}-1\right) \\
& +\frac{1}{\sum h_{t}^{2}} \sum_{t} \sum_{s \neq t} h_{t} h_{s} \epsilon_{t} \epsilon_{s} \cos (s-t) \lambda_{j p} .
\end{aligned}
$$

With $\gamma_{h}=\lim _{n \rightarrow \infty} \sum h_{t}^{2} / n, 0<\gamma_{h}<\infty$, the right hand side of (15) is

$$
\frac{1}{\sum h_{t}^{2}}\left\{\frac{1}{n} \sum_{t=1}^{n}\left(h_{t}^{2} \epsilon_{t}^{2}-\gamma_{h}\right)+\gamma_{h}-\frac{\sum h_{t}^{2}}{n}\right\},
$$


which is $o_{p}(1)$ because $\frac{1}{n} \sum_{t=1}^{n} h_{t}^{2} \epsilon_{t}^{2}-\gamma_{h} \rightarrow_{p} 0$ from Theorem 1 of Heyde and Seneta (1972) (cf. the proof of Theorem 1 in Robinson, 1995b), since the triangular array $h_{t} \epsilon_{t}$ satisfies the same regularity conditions as $\epsilon_{t}$ because $\left|h_{t}\right| \leq 1$ and $\gamma_{h}=\lim _{n \rightarrow \infty} \frac{1}{n} \sum_{t=1}^{n} E\left[h_{t}^{2} \epsilon_{t}^{2} \mid \mathcal{F}_{t-1}\right]>0$ a.s.

Next, we consider the contribution of (16). For $0<r<n /(2 p)$ and $h(j, k)=\sum_{t=1}^{n} \sum_{s=1}^{n} h_{t}^{2} h_{s}^{2}$ $\times \cos (s-t) \lambda_{j p} \cos (s-t) \lambda_{k p}$,

$$
\begin{aligned}
E\left(\sum_{t} \sum_{s \neq t} h_{t} h_{s} \epsilon_{t} \epsilon_{s} \sum_{j=1}^{r} \cos (s-t) \lambda_{j p}\right)^{2} & =2 \sum_{t} \sum_{s \neq t} h_{t}^{2} h_{s}^{2}\left(\sum_{j=1}^{r} \cos (s-t) \lambda_{j p}\right)^{2} \\
& =2 \sum_{j=1}^{r} \sum_{k=1}^{r}\left(h(j, k)-\sum_{t} h_{t}^{4}\right) .
\end{aligned}
$$

Then, changing variables and using trigonometric identities (see also Lemma 7 of Velasco, 1999b),

$$
\begin{aligned}
h(j, k) & =\sum_{t=1}^{n} \sum_{s=1-t}^{n-t} h_{t}^{2} h_{s+t}^{2} \cos s \lambda_{j p} \cos s \lambda_{k p} \\
& =\frac{1}{2} \sum_{t=1}^{n} \sum_{s=1-t}^{n-t} h_{t}^{2} h_{s+t}^{2}\left(\cos s \lambda_{(j+k) p}+\cos s \lambda_{(j-k) p}\right) \\
& =\sum_{t=1}^{n} \sum_{s=1}^{n-t} h_{t}^{2} h_{s+t}^{2}\left(\cos s \lambda_{(j+k) p}+\cos s \lambda_{(j-k) p}\right)
\end{aligned}
$$

Using Lemmas 8 and 9, this is

$$
\begin{gathered}
\frac{1}{2}\left(\left[\sum_{1}^{n} h_{t}^{2} \cos t \lambda_{(j+k) p}\right]^{2}+\left[\sum_{1}^{n} h_{t}^{2} \cos t \lambda_{(j-k) p}\right]^{2}+O(n)\right) \\
=O\left(n^{2}\left[|j+k|^{-2 p}+|j-k|^{-2 p}\right]+n\right),
\end{gathered}
$$

so (17) is $O\left(n^{2} r+r^{2} n\right), 1 \leq r \leq n /(2 p)$. Therefore (14) holds for $p>1$ and the Lemma follows now as when $p=1$ using (13).

Proof of Lemma 2. Follows from the pointwise convergence in Lemma 1 and an equicontinuity argument using the compactness of $\Theta_{1}$, and the differentiability of $\psi(\lambda, \theta)$ with respect to $\theta$ (cf. Hannan, 1973).

Proof of Lemma 3. We consider only the scalar case, the argument for the vector case being identical but notationally more complex, since the stochastic argument, $I^{p}\left(\lambda_{j}\right)$, is scalar. We follow the same procedure as in the proof of Lemma 1.

No tapering $[p=1]$. Using the second moments of the periodogram and Robinson's (1995b) pp. 1648-51 procedure, in Lemma 1 in Velasco (1999b), $1 \leq r \leq n / 2, d \in\left(-\frac{1}{2}, 1\right)$ we find that

$$
\begin{gathered}
\sum_{j=1}^{r}\left\{\frac{I\left(\lambda_{j}\right)}{f\left(\lambda_{j}\right)}-2 \pi I^{\epsilon}\left(\lambda_{j}\right)\right\} \\
=O_{p}\left(r^{1 / 3}(\log n)^{2 / 3}+r^{1 / 2} n^{-1 / 4}+r^{1 /\left(5-4 d_{o}\right)}(\log r)^{2 /\left(5-4 d_{o}\right)}\right.
\end{gathered}
$$




$$
\left.+r^{2 d_{o}-1} \log r+n^{-1 / 2} r^{\left(1+d_{o}\right) / 2}(\log n)^{5 / 4}+n^{-1 / 4} r^{d_{o}}(\log r)^{1 / 2}\right) .
$$

Now, using the same arguments and $\psi(\lambda)=O\left(f^{-1}(\lambda)|\lambda|^{-\delta}\right)$ as $\lambda \rightarrow 0, H_{n}-H_{n}^{\epsilon}$ is

$$
\begin{gathered}
O_{p}\left(n ^ { \delta - 1 } \left[n^{1 / 3}(\log n)^{1 / 3}+n^{1 / 4}+n^{1 /\left(5-4 d_{o}\right)}(\log n)^{2 /\left(5-4 d_{o}\right)}\right.\right. \\
\left.\left.+n^{2 d_{o}-1} \log n+n^{d_{o} / 2}(\log n)^{5 / 4}+n^{d_{o}-1 / 4}(\log n)^{1 / 2}\right]\right)
\end{gathered}
$$

which is $o_{p}\left(n^{-1 / 2}\right)$ if $d_{o}<3 / 4$.

Tapering $[p>1]$. In Velasco (1999b) it is obtained that, $\beta>1,1 \leq r<n /(2 p)$,

$$
\begin{aligned}
& \sum_{j=1}^{r}\left(\frac{I^{p}\left(\lambda_{j p}\right)}{f\left(\lambda_{j p}\right)}-2 \pi I^{p, \epsilon}\left(\lambda_{j p}\right)\right) \\
= & O_{p}\left(r^{1-\beta / 2}+\log r+r^{d_{o}-p+1}(\log n)^{1 / 2}\right),
\end{aligned}
$$

so adapting the proof, $H_{n}-H_{n}^{\epsilon}=O_{p}\left(n^{\delta-1}\left[\log n+n^{1-\beta / 2}+n^{d_{o}-p+1}(\log n)^{1 / 2}\right]\right)=o_{p}\left(n^{-1 / 2}\right)$ if $\beta>1, p>d_{o}+1 / 2$.

We now consider simultaneously the situations $p=1$ and $p>1$, but stressing the tapering situation, the untapered case being simpler since many bounds are exactly zero due to the exact orthogonality of the sine and cosine functions. We have for $g=f \psi$, that $\int_{-\pi}^{\pi} g(\lambda) d \lambda=0$, so $(2 \pi p / n) \sum_{j(p)} g\left(\lambda_{j}\right)=O\left(n^{\delta-1}\right)=o\left(n^{-1 / 2}\right)$, from Lemma 5 , and $H_{n}^{\epsilon}=H_{n}^{\star}+o_{p}\left(n^{-1 / 2}\right)$, with

$$
H_{n}^{\star}=\frac{4 \pi p}{n} \sum_{j=1}^{n^{*}} g\left(\lambda_{j p}\right)\left\{2 \pi I^{p, \epsilon}\left(\lambda_{j p}\right)-1\right\} .
$$

Then $E\left[H_{n}^{\star}\right]=0$ and $\sqrt{n / p} H_{n}^{\star}=\sum_{t=1}^{n} z_{t}$, where $z_{t}=h_{t} \epsilon_{t} \sum_{s=1}^{t-1} h_{s} \epsilon_{s} c_{t-s}$ is a martingale difference sequence and $c_{s}=4 \pi\left(\sum h_{t}^{2}\right)^{-1} \sqrt{p / n} \sum_{j=1}^{n^{*}} g\left(\lambda_{j p}\right) \cos \left(s \lambda_{j p}\right)$. Now we follow the same method of proof as in Robinson (1995b), Theorem 2, to show the asymptotic normality of $H_{n}^{*}$. First we need to show that

$$
\sum_{1}^{n} E\left[z_{t}^{2} \mid \mathcal{F}_{t-1}\right] \rightarrow_{p} \Phi_{p} 4 \pi \int_{-\pi}^{\pi} g^{2}(\lambda) d \lambda
$$

The left hand side is

$$
\sum_{t=2}^{n} h_{t}^{2} \sum_{s=1}^{t-1} h_{s}^{2} \epsilon_{s}^{2} c_{t-s}^{2}+\sum_{t=1}^{n} h_{t}^{2} \sum_{s=1}^{t-1} \sum_{r \neq s}^{t-1} h_{s} \epsilon_{s} h_{r} \epsilon_{r} c_{t-s} c_{t-r} .
$$

The first term in (19) is

$$
\sum_{t=1}^{n-1} h_{t}^{2}\left(\epsilon_{t}^{2}-1\right) \sum_{s=1}^{n-t} h_{s+t}^{2} c_{s}^{2}+\sum_{t=1}^{n-1} h_{t}^{2} \sum_{s=1}^{n-t} h_{s+t}^{2} c_{s}^{2}=B_{1}+B_{2}
$$

say. $B_{1}$ is $o_{p}(1)$, since it has zero mean and variance $O\left(\sum_{1}^{n-1}\left(\sum_{s=1}^{n-t} c_{s}^{2}\right)^{2}\right)$, and from Robinson (1995b) (top of p. 1646), we obtain using summation by parts, for any $\delta>0, c_{s}=c_{n-s}$, and

$$
\left|c_{s}\right| \leq C \mid n^{-3 / 2} \sum_{j=1}^{n^{*}}\left(g\left(\lambda_{j p}\right)-g\left(\lambda_{(j+1) p}\right)\right) \sum_{\ell=1}^{j} \cos s \lambda_{\ell p}
$$




$$
\begin{aligned}
+n^{-3 / 2} g\left(\lambda_{p n^{*}}\right) \sum_{j=1}^{n^{*}} \cos s \lambda_{j p} \mid \\
\leq C n^{-1 / 2} s^{-1}\left\{n^{\delta} \sum_{j=1}^{n^{*}-1} j^{-1-\delta}+1\right\}=O\left(n^{\delta-1 / 2} s^{-1}\right),
\end{aligned}
$$

for $1 \leq s \leq n / 2$, so $\sum_{s=1}^{n} c_{s}^{2}=O\left(n^{2 \delta-1}\right)$. By Lemma $6 B_{2}=V_{n} \sim \Phi_{p} 4 \pi \int_{-\pi}^{\pi} g^{2}(\lambda) d \lambda$.

The second term in (19) can be shown to be $o_{p}(1)$, using the same argument (see also Velasco, 1999b, Lemma 6), because it has zero mean and variance

$$
\begin{aligned}
& 2 \sum_{t=2}^{n} h_{t}^{2} \sum_{u=2}^{n} h_{u}^{2} \sum_{s}^{\min \{t-1, u-1\}} \sum_{r \neq s} h_{s}^{2} h_{r}^{2} c_{t-r} c_{t-s} c_{u-r} c_{u-s} \\
& =2 \sum_{t=2}^{n} h_{t}^{4} \sum_{s} \sum_{r \neq s} h_{s}^{2} h_{r}^{2} c_{t-r}^{2} c_{t-s}^{2}+4 \sum_{t=3}^{n} h_{t}^{2} \sum_{u=2}^{t-1} h_{u}^{2} \sum_{s}^{u-1} \sum_{r \neq s}^{u-1} h_{s}^{2} h_{r}^{2} c_{t-r} c_{t-s} c_{u-r} c_{u-s},
\end{aligned}
$$

because the weights $\left\{h_{t}\right\}$ are symmetric around $\lfloor n / 2\rfloor$. Using the bounds for $c_{s}$ and $\sum_{1}^{n} c_{s}^{2}$, and since $\sup _{t}\left|h_{t}\right| \leq 1$, the first term is $O\left(n^{2 \delta-1}\right)=o(1)$ and the second has absolute value bounded by

$$
\begin{aligned}
& 4 \sum_{t=3}^{n} \sum_{u=2}^{t-1}\left(\sum_{s}^{u-1} c_{t-r}^{2} \sum_{r \neq s}^{u-1} c_{u-r}^{2}\right) \\
& \leq 4\left(\sum_{1}^{n} c_{t}^{2}\right)\left(\sum_{t=3}^{n} \sum_{u=2}^{t-1} \sum_{r=t-u+1}^{t-1} c_{r}^{2}\right) \\
& \leq 4\left(\sum_{1}^{n} c_{t}^{2}\right)\left(2 n \sum_{j=1}^{n^{*}} j c_{j}^{2}\right),
\end{aligned}
$$

and this is $O\left(n^{4 \delta-1} \log n\right)=o(1)$. Thus (18) is proved.

Finally we need to show that $\sum_{1}^{n} E\left[z_{t}^{2} I\left(\left|z_{t}\right|>\rho\right)\right] \rightarrow 0$ for all $\rho>0$, for which we can check the sufficient condition $\sum_{1}^{n} E\left[z_{t}^{4}\right] \rightarrow 0$. Following Robinson (1995b), $\sum_{1}^{n} E\left[z_{t}^{4}\right] \leq C n\left(\sum_{1}^{n} c_{s}^{2}\right)^{2}=$ $O\left(n^{2 \delta-1}\right)=o(1)$ and the central limit theorem follows.

Proof of Lemma 4. For $\varepsilon>0, \psi(\lambda)=|\lambda|^{2\left(d_{o}+\varepsilon-1 / 2\right)}=O\left(f^{-1}(\lambda)|\lambda|^{2 \varepsilon-1}\right)$ satisfies the conditions of Lemma 1 , with $\delta=2 \varepsilon-1,0<\delta<1$.

Proof of Lemma 5. This follows from the discussion in Robinson (1994a), p. 75.

Proof of Lemma 6. First, using trigonometric identities we have that $V_{n}$ is

$$
\begin{aligned}
& 4(4 \pi)^{2} \frac{p}{n} \sum_{j=1}^{n^{*}} \sum_{k=1}^{n^{*}} g\left(\lambda_{j p}\right) g\left(\lambda_{k p}\right)\left(\sum_{1}^{n} h_{t}^{2}\right)^{-2} \\
& \times \frac{1}{2} \sum_{t=1}^{n-1} h_{t}^{2} \sum_{s=1}^{n-t} h_{s+t}^{2}\left\{\cos s \lambda_{(j+k) p}+\cos s \lambda_{(j-k) p}\right\}
\end{aligned}
$$

and using Lemma 9 and $h\left(\lambda_{j}\right)=\left(\sum_{1}^{n} h_{t}^{2}\right)^{-1} \sum_{1}^{n} h_{t}^{2} \cos t \lambda_{j}$, this is

$$
\frac{(4 \pi)^{2} p}{n} \sum_{j=1}^{n^{*}} \sum_{k=1}^{n^{*}} g\left(\lambda_{j p}\right) g\left(\lambda_{k p}\right)\left\{h^{2}\left(\lambda_{(j+k) p}\right)+h^{2}\left(\lambda_{(j-k) p}\right)\right\},
$$


plus a term of smaller order of magnitude which is $O\left(n^{-3} \sum_{j} \sum_{k} g\left(\lambda_{j p}\right) g\left(\lambda_{k p}\right)\right)=o(1)$. From Lemma $8, h\left(\lambda_{j}\right)=O\left(|j|^{-p}\right)$, and with $g(\lambda)=O\left(|\lambda|^{-\delta}\right)$ for any $\delta>0$, the term in $h^{2}\left(\lambda_{(j+k) p}\right)$ of (20) is

$$
O\left(n^{2 \delta-1} \sum_{j=1}^{n} \sum_{k=1}^{n}(j+k)^{-2 p}\right)=O\left(n^{2 \delta-1} \sum_{j=1}^{n} \sum_{k=1}^{n} j^{-p} k^{-p}\right)
$$

which is $O\left(n^{2 \delta-1}\right)=o(1)$, and can be ignored. The other term with $h^{2}\left(\lambda_{(j-k) p}\right)$ in $(20)$ is, $0 \leq \eta \leq n / 2$,

$$
\begin{aligned}
& (4 \pi)^{2} \frac{p}{n} \sum_{j=1}^{n^{*}} g^{2}\left(\lambda_{j p}\right) \sum_{k:|j-k| \leq \eta} h^{2}\left(\lambda_{(j-k) p}\right) \\
+ & O\left(n^{2 \delta-1} \sum_{j=1}^{n^{*}} j^{-\delta} \sum_{k:|j-k|>\eta}|j-k|^{-2 p}\right) \\
+ & n^{-1} \sum_{j=1}^{n^{*}} \sum_{k: 1 \leq|j-k| \leq \eta}\left|g\left(\lambda_{j p}\right)\right| \\
& \times O\left(\left|\sup _{|j-k| \leq \eta}\right| g\left(\lambda_{j p}\right)-g\left(\lambda_{k p}\right)|| j-\left.k\right|^{-2 p}\right)
\end{aligned}
$$

by Lemma 8. This is, using that $\sup _{|j-k| \leq \eta}\left|g\left(\lambda_{j p}\right)-g\left(\lambda_{k p}\right)\right|=O\left(\left|g\left(\lambda_{k p}\right)\right|\left|\lambda_{k}\right|^{-1}\left|\lambda_{k}-\lambda_{j}\right|\right.$ $\left.+\left|g\left(\lambda_{j p}\right)\right|\left|\lambda_{j}\right|^{-1}\left|\lambda_{k}-\lambda_{j}\right|\right)$,

$$
\begin{aligned}
(4 \pi)^{2} & \frac{p}{n} \sum_{j(p)} g^{2}\left(\lambda_{j}\right) \sum_{k=0, p, 2 p}^{n-p} h^{2}\left(\lambda_{k p}\right) \\
+ & O\left(n^{\delta} \eta^{1-2 p}\right)+O\left(n^{2 \delta-1} \sum_{j=1}^{n^{*}} g^{2}\left(\lambda_{j p}\right) \sum_{k>\eta}^{n} k^{-2 p}\right) \\
& +O\left(n^{2 \delta-1} \sum_{j=1}^{n} j^{-1-2 \delta} \sum_{k=1+j}^{j+\eta}|j-k|^{1-2 p}\right) \\
& +O\left(n^{2 \delta-1}\left\{\sum_{j=1}^{2 \eta}+\sum_{j=1+2 \eta}^{n}\right\} j^{-\delta} \sum_{k=1+j}^{j+\eta} k^{-1-\delta}|j-k|^{1-2 p}\right) \\
= & \Phi_{p} 4 \pi \int_{-\pi}^{\pi} f^{2}(\lambda) \psi^{2}(\lambda) d \lambda+O\left(n^{2 \delta} \eta^{1-2 p}\right) \\
+ & O\left(n^{2 \delta-1}+n^{2 \delta-1} \eta^{1-\delta}+n^{\delta} \eta^{-1-\delta}+n^{\delta} \eta^{1-2 p}\right),
\end{aligned}
$$

all the error terms being $o(1)$ on choosing, e.g., $\eta \sim \sqrt{n}$, with $p>1$.

Proof of Lemma 7. By the definition of $\theta_{o}$ and $\widehat{\theta}, \int k_{\theta}^{-1}\left(\lambda ; \theta_{o}\right) f(\lambda) d \lambda=(2 \pi p / n) \sum k_{\theta}^{-1}\left(\lambda_{j} ; \widehat{\theta}\right) I^{p}\left(\lambda_{j}\right)$ $=0$. It follows by the mean value theorem that $\widehat{\theta}-\theta_{o}=\widetilde{\Xi}_{n}^{-1}\left(b_{n}-\zeta\right)$, where $\zeta=(2 \pi p / n) \sum k_{\theta}^{-1}\left(\lambda_{j} ; \theta_{o}\right)$ $\left[I^{p}\left(\lambda_{j}\right)-f\left(\lambda_{j}\right)\right]=O_{p}\left(n^{-1 / 2}\right)$ from Lemma $3, b_{n}=(2 \pi p / n) \sum k_{\theta}^{-1}\left(\lambda_{j} ; \theta_{o}\right) I^{p}\left(\lambda_{j}\right)-\int k_{\theta}^{-1}\left(\lambda ; \theta_{o}\right) f(\lambda) d \lambda$, and the $\ell$ th row of $\widetilde{\Xi}_{n}$ is the $\ell$ th row of the matrix $\Xi_{n}(\theta)=(2 \pi p / n) \sum k_{\theta \theta^{\prime}}^{-1}\left(\lambda_{j} ; \theta\right) I^{p}\left(\lambda_{j}\right)$ evaluated at $\widetilde{\theta}_{\ell}$, which is in the line segment between $\theta_{o}$ and $\widehat{\theta}$. Thus $\widehat{\theta}-\theta_{o}=-\Xi_{o}^{-1} \zeta-a_{n} \zeta+\widetilde{\Xi}_{n}^{-1} b_{n}$, with $a_{n}=\widetilde{\Xi}_{n}^{-1}-\Xi_{o}^{-1}$. The lemma follows if $a_{n}=o_{p}(1),\left\|\widetilde{\Xi}_{n}^{-1}\right\|=O_{p}(1)$ and $b_{n}=o_{p}\left(n^{-1 / 2}\right)$. 
First we bound $a_{n}:\left\|a_{n}\right\| \leq\left\|\widetilde{\Xi}_{n}^{-1}\right\|\left\|\Xi_{o}^{-1}\right\|\left\|\Xi_{o}-\widetilde{\Xi}_{n}\right\|$. From Assumption $9\left\|\Xi_{o}^{-1}\right\|<\infty$ and under the conditions of the lemma $\widetilde{\theta}_{\ell} \rightarrow_{p} \theta_{o}$, so using the continuity of the elements of $\Xi_{n}^{-1}(\theta)$ with respect to $\theta$ (to substitute $\widetilde{\theta}_{\ell}$ by $\theta$ ), the differentiability in $\lambda$ of the second derivatives of $k^{-1}$ for Lemma 1 (to substitute $f$ for $I^{p}$ ), and approximating sums by integrals with Lemma 5 , we can show the elementwise convergence in probability to 0 of $\Xi_{o}-\widetilde{\Xi}_{n}$, and we obtain for $n$ large enough that $\left\|\widetilde{\Xi}_{n}\right\|<\infty$ with probability approaching 1 and thus $a_{n}=o_{p}(1)$.

The bound for $b_{n}$ follows by the previous argument, using Lemmas 1 and 5 to approximate $I^{p}$ by $f$ and sums by integrals, using the differentiability in $\lambda$ of $k(\lambda ; \theta)$ and $k_{\theta}^{-1}(\lambda ; \theta)$, whose derivatives are $O\left(|\lambda|^{-2 d-1}\right)$ and $O\left(|\lambda|^{2 d-1-\delta}\right)$ as $\lambda \rightarrow 0$, respectively, for some $\delta>0$.

Proof of Lemma 8. Follows using the properties of a taper of order $p$, as in Lemma 1 of Velasco (1999b), and that $\sum_{t=1}^{n} h_{t}^{2} \cos t \lambda_{j}=\int_{-\pi}^{\pi} D_{h}\left(\lambda_{j}-\lambda\right) D_{h}(\lambda) d \lambda$.

Proof of Lemma 9. This is part (B) of Lemma 7 in Velasco (1999b).

\section{References}

Alekseev, V. G. (1996), "Jackson- and Jackson-Vallée Poussin-type kernels and their probability applications," Theory of Probability and its Applications, 41, 137-142.

Beran, J. (1994), Statistics for Long-Memory Processes, New York: Chapman \& Hall.

Beran, J. (1995), " Maximum likelihood estimation of the differencing parameter for invertible short- and long-memory ARIMA models," Journal of the Royal Statistical Society, Series B, 57, 659-672.

Beran, J., R.J. Bhansali and D. Ocker (1998), "On unified model selection for stationary and nonstationary short- and long-memory autoregressive processes," Biometrika, 85, 921-934.

Bloomfield, P. (1973), "An exponential model for the spectrum of a scalar time series," Biometrika, 60, 217-226.

Box, G. E. and G. M. Jenkins (1976), Time Series Analysis: Forecasting and Control, San Francisco: Holden Day.

Dahlhaus, R. (1985), "Asymptotic normality of spectral estimates," Journal of Multivariate Analysis, 16, 412-431.

Dahlhaus, R. (1988), "Small sample effects in time series analysis: a new asymptotic theory and a new estimate," Annals of Statistics, 16, 808-841.

Dahlhaus, R. (1989), "Efficient parameter estimation for self-similar processes," Annals of Statistics, 14, 517-532.

Davies, R. B. and D. S. Harte (1987), "Tests for the Hurst effect," Biometrika, 74, 95-101.

Fox, R. and M. S. Taqqu (1986), "Large-sample properties of parameter estimates for strongly dependent stationary Gaussian times series," Annals of Statistics, 14, 517-532.

Geweke, J. and S. Porter-Hudak (1983), "The estimation and application of long memory time series models," Journal of Time Series Analysis, 4, 221-238. 
Giraitis, L. and D. Surgailis (1990), "A central limit theorem for quadratic forms in strongly dependent linear variables and its application to asymptotic normality of Whittle's estimate," Probability Theory and Related Fields, 86, 87-104.

Hannan, E. J. (1973), "The asymptotic theory of linear time series models," Journal of Applied Probability, 10, 130-145.

Haslett, J. and Raftery, A. E. (1989), "Space-time modeling with long-memory dependence: assessing Ireland's wind power resource (with discussion)," Applied Statistics, 38, 1-50.

Hauser, M. A. (1999), "Maximum likelihood estimates for ARFIMA models: A Monte Carlo study," Journal of Statistical Planning and Inference, 80, 229-255.

Hosoya, Y. (1996), "The quasi-likelihood approach to statistical inference on multiple timeseries with long-range dependence," Journal of Econometrics, 73, 217-236.

Hosoya, Y. and M. Taniguchi (1982), "A central limit theorem for stationary processes and the parameter estimation of linear processes," Annals of Statistics, 10, 132-153.

Hurvich, C. M. and B. K. Ray (1995), "Estimation of the memory parameter for nonstationary or noninvertible fractionally integrated processes," Journal of Time Series Analysis, 16, $17-42$.

Ling, S. and W. K. Li (1997), "On fractionally integrated autoregressive moving-average time series models with conditional heteroscedasticity," Journal of the American Statistical Association, 92, 1184-1194.

Robinson, P. M. (1978), "Alternative models for stationary stochastic processes," Stochastic Processes and their Applications, 8, 141-152.

Robinson, P. M. (1986), "On the errors-in-variables problem for time series," Journal of Multivariate Analysis, 19, 240-250.

Robinson, P. M. (1994a), "Time series with strong dependence", in Advances in Econometrics. Sixth World Congress, Vol. 1, C.A. Sims., ed., pp. 47-95, Cambridge: Cambridge University Press.

Robinson, P. M. (1994b), "Efficient tests of nonstationary hypotheses," Journal of the American Statistical Association, 89, 1420-1437.

Robinson, P. M. (1995a), "Log-periodogram regression of time series with long range dependence," Annals of Statistics, 23, 1048-1072.

Robinson, P. M. (1995b), "Gaussian semiparametric estimation of long range dependence," Annals of Statistics, 23, 1630-1661.

Solo, V. (1984), "The order of differencing in ARMA models," Journal of the American Statistical Association, 79, 916-921.

Solo, V. (1989), "Asymptotics of quadratic forms for time series", unpublished manuscript.

Solo, V. (1992), "Intrinsic random functions and the paradox of 1/f noise", SIAM Journal of Applied Mathematics, 52, 270-291. 
Tukey, J. W. (1967), "An introduction to the calculation of numerical spectrum analysis," in Advanced Seminar on Spectral Analysis of Time Series, ed. B. Harris, pp. 25-46, New York: Wiley.

Velasco, C. (1999a), "Non-stationary log-periodogram regression," Journal of Econometrics, 91, 325-371, 1999.

Velasco, C. (1999b), "Gaussian semiparametric estimation for non-stationary time series," Journal of Time Series Analysis, 20, 87-127.

Zhurbenko, I. G. (1979), "On the efficiency of estimates of a spectral density," Scandinavian Journal of Statistics, 6, 49-56. 